\title{
Nonlinear Normal Modes in a Two-Stage Isolator Using a Modified Finite-Element Galerkin Method
}

\author{
Cheng Li $(\mathbb{D})$ and Hongguang $\mathrm{Li}$ (iD) \\ Institute of Vibration, Shock and Noise, State Key Laboratory of Mechanical System and Vibration, \\ Shanghai Jiao Tong University, Shanghai 200240, China
}

Correspondence should be addressed to Hongguang Li; hgli@sjtu.edu.cn

Received 14 October 2020; Revised 15 March 2021; Accepted 4 April 2021; Published 29 May 2021

Academic Editor: Giuseppe Ruta

Copyright $(92021$ Cheng Li and Hongguang Li. This is an open access article distributed under the Creative Commons Attribution License, which permits unrestricted use, distribution, and reproduction in any medium, provided the original work is properly cited.

\begin{abstract}
A modified Galerkin method is proposed to approximate the nonlinear normal modes in a new type of a two-stage isolator. Besides the displacement of payload and the force transmissibility of this typical nonlinear dynamic system, the nonlinear normal modes defined as invariant manifolds can provide more information about the nonlinear coupling between the system components when periodic motions corresponding to the normal modes of the system occur. The presented approach applies a combination of finite-element discretization and Fourier series expansion for the approximate invariant manifolds. A Galerkin projection of the governing equations for the approximate invariant manifolds yields a set of nonlinear algebraic equations in expansion coefficients, which can be solved numerically with a general choice of zero as initial guess for the cases in this work. The resultant approximate solutions for the invariant manifolds can accurately describe the nonlinear interactions between system components in periodic motions of the specific nonlinear normal modes. In addition, one can solve the invariant manifolds for an annular domain of interest directly by this method, without considering other domain that includes the origin of phase space.
\end{abstract}

\section{Introduction}

Linear vibration isolators can be used when their natural frequencies are well below the excitation frequency. An expected small natural frequency can be obtained from an isolation structure with low stiffness which also leads to an undesirable large static displacement. One kind of nonlinear isolator with a high static but low dynamic stiffness is proposed to resolve this issue, which is represented by a configuration with lateral and vertical springs [1]. A comprehensive review about nonlinear passive vibration isolators is given by Ibrahim [2]. This kind of geometric design for stiffness nonlinearity is exploited in a class of two-stage nonlinear isolator $[3,4]$ which can have a desirable small static deflection as well as a smaller transmissibility in the frequency range of isolation compared with the corresponding linear two-stage isolator. Two versions of this class of two-stage nonlinear isolator are proposed. One is that both the payload of upper stage and the additional mass of lower stage are attached to the ground by nonlinear isolating devices, respectively (two sets of springs and dampers) [3] (see Figure 1(a)). In the other one [4] (see Figure 1(b)), the payload platform, referred as upper stage, is only linked to an additional mass platform through a nonlinear isolating device (a set of springs and dampers) and has no direct connection with the ground. The additional mass platform, referred as lower stage, is connected to the ground by another set of springs and dampers. As a complement to this class of two-stage isolators, the third configuration of nonlinear isolator is considered in this paper. In this new version, the payload and the ground are connected directly through an upper-stage isolating device while the additional mass is only attached to the payload platform by a nonlinear lower-stage isolating device, having no direct connection with the ground (see Figure 1(c)).

Besides the forced transmissibilities or displacementfrequency responses, this work also focuses on the nonlinear forced resonance of this new type two-stage isolator 


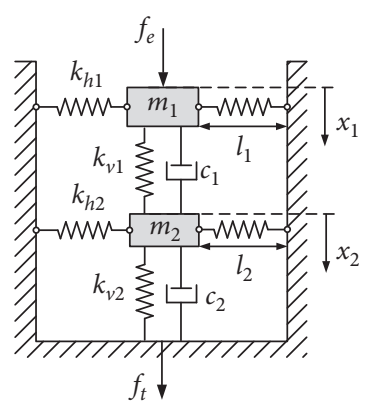

(a)

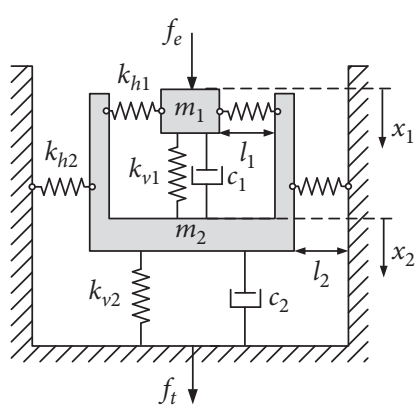

(b)

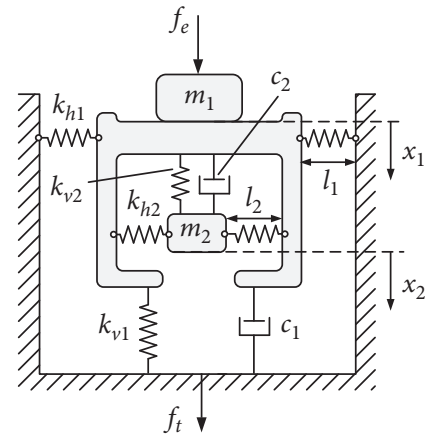

(c)

Figure 1: Schematic diagrams of three types of nonlinear two-stage isolators. The payload is set on the upper stage in each type: (a) type I isolator of which two stages are both connected to the foundation [3]; (b) type II isolator of which upper stage has no direct connection with the foundation [4]; (c) type III isolator of which lower stage only connects with the upper one.

for the cases where the frequencies of harmonic excitations just falls in or passes through the primary resonant frequency range. As a typical nonlinear multidegree-offreedom system, it exhibits noticeable nonlinear characteristics of primary resonance which can be depicted by a concept known as nonlinear normal modes (NNMs) of the underlying conservative systems [5]. Shaw and Pierre defined an NNM as a two-dimensional invariant manifold in phase space [6-8] according to the center manifold theorem [9]. This manifold is a subset of phase space where orbits of the dynamic system which start out from the manifold remain in it for all time. This is also shown in this work. The forced resonances in nonlinear systems occur in the neighborhoods of corresponding NNMs of the underlying conservative systems $[5,10]$. Hence, the NNMs can provide a valuable tool for exhibiting the forced response structure of the resonance which is a significant feature of engineering importance.

In autonomous conservative systems, a family of periodic oscillations which lie on the NNM invariant manifolds can be computed numerically using shooting method and algorithms for the continuation of periodic solutions [11]. These numerical periodic orbits as reference solutions are compared with the following approximations of invariant manifolds of the nonlinear vibrating system to verify the proposed method's validity. Different techniques for computing NNMs of vibrating systems are reviewed by G. Kerschen et al. [10]. For the NNMs defined in terms of invariant manifolds, an asymptotic expansion can be applied to build the NNMs approximately $[12,13]$, but the asymptotic solutions are accurate only for small-amplitude motions in a neighborhood of the original equilibrium of the system. With a Galerkin projection method [14], an approximation of the NNMs defined as invariant manifolds can be solved accurately for a wide range of nonlinear vibrating systems. For the concerned NNMs of the new twostage isolator, considering the strong nonlinearity of this system and the resulting strongly coupled algebraic equations from the standard Galerkin procedure, a modified finite-element Galerkin approach is proposed to simplify the nonlinear algebraic equations and to facilitate the solving of the partial differential equations which govern the NNM invariant manifolds.

\section{A New Type of a Two-Stage Nonlinear Isolator}

Figure 1 displays the diagrams of three types of nonlinear two-stage isolators. The classification depends on the typical configurations of connections between the stages (upper or lower stage) and the foundation. Specifically, the lower stage with an additional mass in type III is only linked to the upper stage with payload via stiffness and damping components, without any direct connection with the foundation. As a comparison, the upper stage with payload in type II has merely connections with the lower stage and has no direct connection with the foundation. In type I, both stages are linked to the foundation.

Type III's isolation characteristics are of interest in this section when the stage of payload is subjected to harmonic force excitations, which could be measured by force transmissibilities or displacement-frequency responses of the payload. The isolating structures in both stages may have a hardening or softening stiffness nonlinearity which is common to a lot of classical nonlinear isolators $[2,15]$ and can be simplified to polynomial stiffness as in a few new isolators [16-22]. In each stage model, there are two auxiliary lateral springs with stiffness $k_{h 1}$ (or $k_{h 2}$ ) such as a vertical spring with stiffness $k_{v 1}$ (or $k_{v 2}$ ) and a vertical linear damper with coefficient $c_{1}$ (or $c_{2}$ ) as shown in Figure 1. The vertical springs support the payload or additional mass while the lateral springs provide negative stiffness to adjust the natural frequency of the system. The force-deflection characteristic for the set of springs has been given in detail in reference [1]. The equations of motion for the system subjected to harmonic force excitation $F_{e} \cos (\omega t)$ are given by 


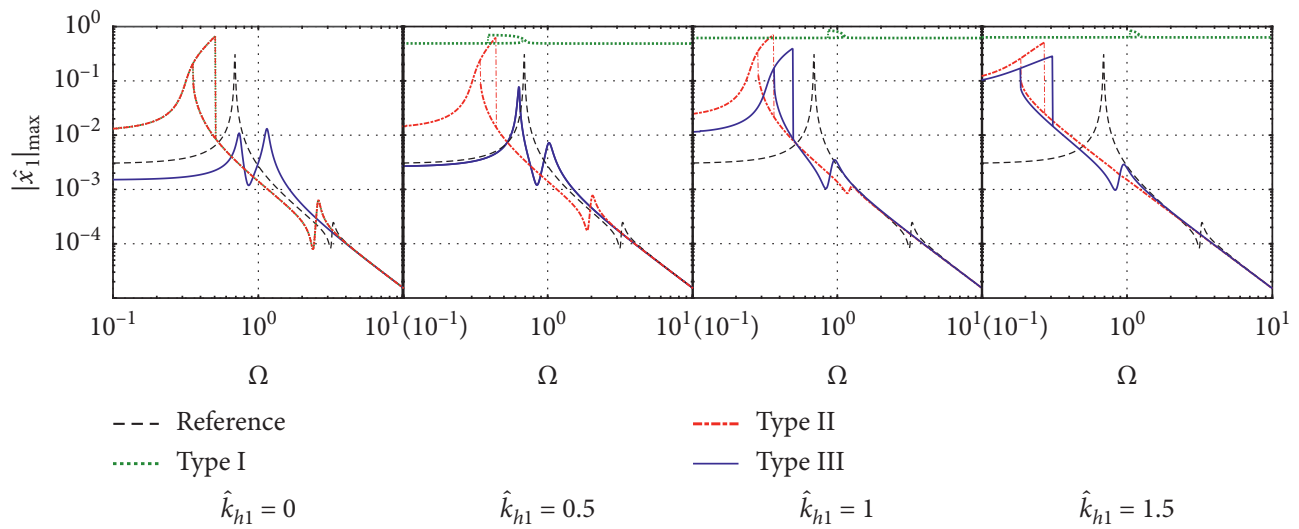

(a)

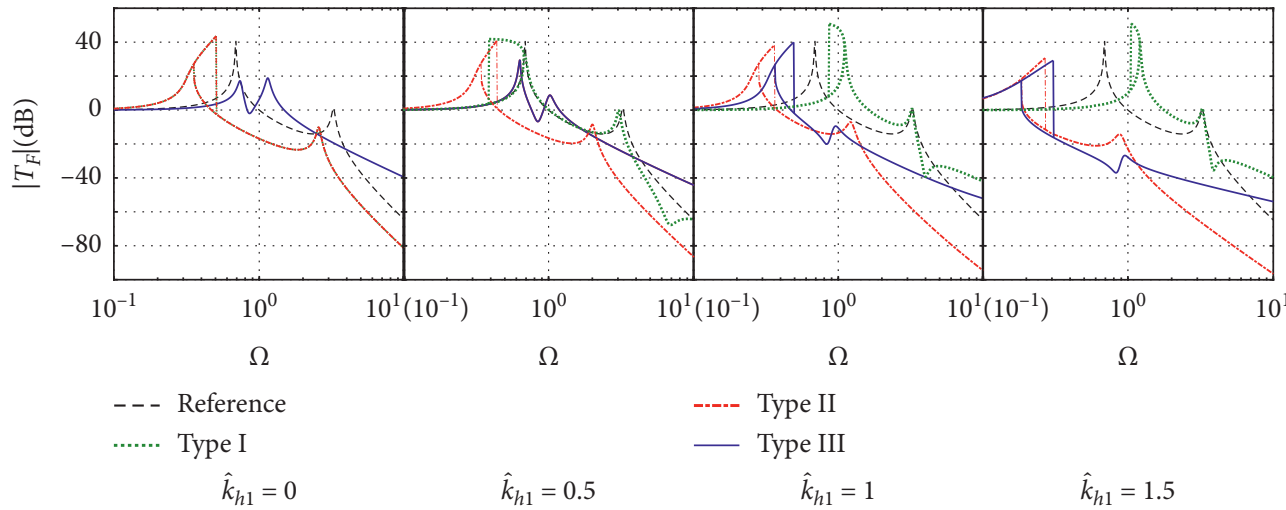

(b)

FIGURE 2: Top plots: displacement amplitude-frequency responses for the payloads in four types of two-stage isolators, for $\mu=0.2, \zeta_{1}=\zeta_{2}=0.01$, $\widehat{k}_{h 2}=\widehat{k}_{v 2}=1.0, \widehat{l}=0.7$, and $F=0.0015$. Bottom plots: magnitudes of the force transmissibility in four types of two-stage isolators. Black dashed lines, linear two-stage isolator (corresponding to a model as shown in Figure 1(a) with $\widehat{k}_{h 1}=\widehat{k}_{h 2}=0$; red dashed-dotted lines, type I; green dotted lines, type II; blue solid lines, type III. The responses and the force transmissibility of three nonlinear isolators are compared for different values of the upper lateral stiffness $\widehat{k}_{h 1}: \widehat{k}_{h 1}=0, \widehat{k}_{h 1}=0.5, \widehat{k}_{h 1}=1.0$, and $\widehat{k}_{h 1}=1.15$ (if $\widehat{k}_{h 1}$ reaches a value beyond 1.15 when $\widehat{k}_{v 2}=1.0$ and $\widehat{l}=0.7$, the nonlinear isolator systems can easily become bi-stable for small changes of stiffness, which is undesirable and is not considered in this work).

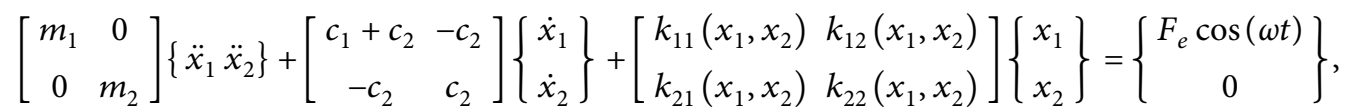

where $x_{1}$ and $x_{2}$ are the displacement of the upper stage with payload $m_{1}$ and the one of the low-stage $m_{2}$, respectively, and their equilibrium positions are set such that the lateral springs $k_{h 1}$ and $k_{h 2}$ are both horizontal when the payload is placed on the upper stage statically. In equation (1), $k_{11}\left(x_{1}, x_{2}\right)=k_{v 1}+2 k_{h 1} . \quad\left(1-\left(l_{o 1} / \sqrt{x_{1}^{2}+l_{1}^{2}}\right)\right)+k_{v 2}+2 k_{h 2}$. $\left.\left(1-\left(l_{o 2} / \sqrt{\left(x_{1}\right.}-x_{2}\right)^{2}+l_{2}^{2}\right)\right), \quad k_{12}\left(x_{1}, x_{2}\right)=k_{21} \quad\left(x_{1}, x_{2}\right)=$ $-k_{v 2}-2 k_{h 2} \cdot\left(1-l_{o 2} / \sqrt{\left(x_{1}-x_{2}\right)^{2}+l_{2}^{2}}\right)$, and $k_{22}\left(x_{1}, x_{2}\right)=$ $k_{v 2}+2 k_{h 2} \cdot\left(1-l_{o 2} / \sqrt{\left(x_{1}-x_{2}\right)^{2}+l_{2}^{2}}\right)$. $l_{1}$ and $l_{2}$ are the lengths of the lateral springs in each stage, respectively, when they are in the horizontal position as shown in Figure $1 . l_{o 1}$ and $l_{o 2}$ are their original lengths, respectively. The restoring forces provided by the isolators in different stages can be approximated by a third-order polynomial if the deflections of the payload and the additional mass are such that $x_{1}<0.2 l_{1}$ and $x_{2}<0.2 l_{2}[3,4]$. Hence, equation (1) can be reduced to two coupled Duffing equations in nondimensional form:

$$
\left[\begin{array}{ll}
1 & 0 \\
0 & \mu
\end{array}\right]\left\{\begin{array}{l}
\ddot{\hat{x}}_{1} \\
\ddot{\hat{x}}_{2}
\end{array}\right\}+2\left[\begin{array}{cc}
\zeta_{1}+\mu \zeta_{2} & -\zeta_{2} \\
-\zeta_{2} & \zeta_{2}
\end{array}\right]\left\{\begin{array}{l}
\dot{\hat{x}}_{1} \\
\dot{\hat{x}}_{2}
\end{array}\right\}+\left[\begin{array}{cc}
\Omega_{1}^{2}+\Omega_{2}^{2} & -\Omega_{2}^{2} \\
-\Omega_{2}^{2} & \Omega_{2}^{2}
\end{array}\right]\left\{\begin{array}{l}
\hat{x}_{1} \\
\hat{x}_{2}
\end{array}\right\}+\left[\begin{array}{cc}
\gamma_{1} & -\gamma_{2} \\
0 & \gamma_{2}
\end{array}\right]\left\{\begin{array}{c}
\hat{x}_{1}^{3} \\
\left(\hat{x}_{1}-\hat{x}_{2}\right)^{3}
\end{array}\right\}=\left\{\begin{array}{c}
F \cos (\Omega \tau) \\
0
\end{array}\right\}
$$


where $x_{s}=\sqrt{l_{o 1}^{2}-l_{1}^{2}}, \hat{x}_{1}=\left(x_{1} / x_{s}\right), \hat{x}_{2}=\left(x_{2} / x_{s}\right), \mu=\left(m_{2} /\right.$ $\left.m_{1}\right), \omega_{n}=\sqrt{\left(k_{v 1} / m_{1}\right)}, \zeta_{1}=\left(c_{1} / 2 m_{1} \omega_{n}\right), \zeta_{2}=\left(c_{2} / 2 m_{2} \omega_{n}\right)$, $\widehat{l}=\left(l_{1} / l_{o 1}\right)=\left(l_{2} / l_{o 2}\right), \quad \widehat{k}_{h 1}=\left(k_{h 1} / k_{v 1}\right), \quad \widehat{k}_{v 2}=\left(\begin{array}{ll}k_{v 2} / & k_{v 1}\end{array}\right)$, $\widehat{k}_{h 2}=\left(k_{h 2} / k_{v 1}\right), \Omega_{1}^{2}=1-2 \cdot((1 / \widehat{l})-1) \cdot \widehat{k}_{h 1}, \Omega_{2}^{2}=\widehat{k}_{v 2}-2$. $((1 / \hat{l})-1) \cdot \widehat{k}_{h 2}, \gamma_{1}=\left(\left(1-\widehat{l}^{2}\right) \widehat{l}^{3}\right) \cdot \widehat{k}_{h 1}, \gamma_{2}=\left(\left(1-\widehat{l}^{2}\right) \widehat{l}^{3}\right) \cdot \widehat{k}_{h 2}$, $\Omega=\left(\omega / \omega_{n}\right), \tau=\omega_{n} t$, and $F=F_{e} /\left(k_{v 1} \cdot x_{s}\right)$.

The geometrical parameters $\hat{l}$ for the lateral springs are assumed to be identical in both stages in this case. Since the connections between the stages and the foundation in type III isolator are different from the connections in type I or in type II, the nonlinear terms in equation (2) are distinct from the ones of the other two systems [3, 4]. All forced responses of the isolator type III, as well as the ones of type I and II for comparison, are obtained at each frequency of harmonic excitations using a fourth-order Runge-Kutta integration scheme instead of the harmonic balance method since the forced responses of nonlinear systems, especially the ones in their primary resonance, are not simplified as harmonic motions in this work.

The amplitude of the force transmissibility [3] for type III isolator is given by

$$
\left|T_{F}\right|=\frac{\left|f_{t}\right|_{\max }}{F}=\frac{\left|2 \zeta_{1} \dot{\hat{x}}_{1}+\Omega_{1}^{2} \hat{x}_{1}+\gamma_{1} \hat{x}_{1}^{3}\right|_{\max }}{F},
$$

which is the ratio between the amplitude of force $f_{t}$ transmitted to the foundation and the amplitude of the harmonic force applied to the payload. The maximum of absolute values of the transmitted force $f_{t}$ for a steady forced motion is taken as its amplitude.

The payloads' displacement amplitude-frequency responses and force transmissibilities for all three types of isolators are presented in Figure 2 for some certain stiffness $\widehat{k}_{h 1}$ of the lateral springs, which are attached to the upper stage including payloads. In addition, the corresponding response and force transmissibility of a linear two-stage isolator with the identical configuration excluding the lateral stiffness components are also shown.

The payload in each isolator system is under a harmonic force excitation with a slowly varying (increasing or decreasing) frequency. The amplitude for the excitation is set to $F=0.0015$, for instance, for all cases where period doubling and chaos [23], which are beyond the scope of this work, and do not arise under the assumption of $x_{1}<0.2 l_{1}$ and $x_{2}<0.2 l_{2}$. The maximum of absolute values of displacement for a steady forced motion, $\left|\widehat{x}_{1}\right|_{\text {max }}$, is taken as its amplitude.

As can be seen in Figure 2, these force transmissibility curves or displacement curves have distinct shapes for different $\widehat{k}_{h 1}$. When $\widehat{k}_{h 1}=0$, type I isolator and type II isolator share the same structure, and the corresponding curves for type I and for type II coincide. As in the case of type I and type II isolator, the stiffness of lateral springs in the upper stage of type III isolator can adjust the degree of its dynamic nonlinearity. As the stiffness $\widehat{k}_{h 1}$ is increased, the linear fundamental natural frequency of isolator type III is reduced, and the range of frequencies for isolation increases even considering the undesirable bending and jump phenomenon of the frequency response curve. For these given system parameters, type I isolator exhibits a softening stiffness nonlinearity when the stiffness $\widehat{k}_{h 1}$ of lateral springs for upper stage is positive, which was not presented in [3]. Type II isolator has smaller force transmissibilities in the high frequency range comparing to the other isolators with the given parameters, and the deflections of payload in type III isolator are smaller than the others when these isolators are excited in their respective primary resonance.

\section{The Computation for NNMs as Invariant Manifolds}

Apart from the forced frequency responses, this typical nonlinear multidegree-of-freedom system also displays some nonlinear features in primary resonance, which can be described approximately by the nonlinear normal modes (NNMs) of its underlying conservative system $[5,10]$. In the following parts, our focus is placed on the NNMs and the calculation of them.

The equations of motion for the system without damping or excitation could be transformed into equations in generalized linear modal coordinates, given as the following standard form:

$$
\left\{\begin{array}{c}
\ddot{\eta}_{1}+\omega_{1}^{2} \eta_{1}+f_{1}\left(\eta_{1}, \eta_{2}, \ldots, \eta_{n}\right)=0 \\
\ddot{\eta}_{2}+\omega_{2}^{2} \eta_{2}+f_{2}\left(\eta_{1}, \eta_{2}, \ldots, \eta_{n}\right)=0 \\
\vdots \\
\ddot{\eta}_{n}+\omega_{n}^{2} \eta_{n}+f_{n}\left(\eta_{1}, \eta_{2}, \ldots, \eta_{n}\right)=0
\end{array}\right.
$$

where $\eta_{i}$ and $\omega_{i}$ for $i=1,2, \ldots, n$ are the $i$ th linear modal coordinate and modal frequency, respectively, and $f_{i}\left(\eta_{1}, \eta_{2}, \ldots, \eta_{n}\right)$ for $i=1,2, \ldots, n$ is the $i$ th nonlinear force which is assumed to be a function of all the linear modal coordinates.

A NNM invariant manifold for this vibratory system is a two-dimensional surface in the phase space which is tangent to the corresponding modal eigenspace of the linearized system at the equilibrium [6-8]. A pair of state variables (e.g., a displacevelocity pair, $x_{1}$ and $\dot{x}_{1}$ in physical coordinates or $\eta_{1}$ and $\dot{\eta}_{1}$ in modal coordinates) could be chosen as the master coordinates for the NNM of interest. The remaining degree of freedoms (e.g., $\eta_{2}, \ldots, \dot{\eta}_{n}$ ) as slave coordinates is constrained to this pair of master coordinates by the NNM manifold surface functions (e.g., $\left.P_{2}\left(\eta_{1}, \dot{\eta}_{1}\right), \ldots, P_{n}\left(\eta_{1}, \dot{\eta}_{1}\right)\right)$.

To illustrate the nonharmonic periodic motion in primary resonance and its evolution around corresponding NNM, Figure 3(b) displays a forced response orbit of a type III isolator in dot-dashed line, for instance, in a phase subspace of physical coordinates $\left(\hat{x}_{1}, \hat{x}_{2}\right)$. This forced nonharmonic periodic motion corresponds to the steady forced responses shown as the square points of the displacement-frequency chart in Figure 3(a), representing a primary resonance under the harmonic force excitation for a specific value of amplitude. The free response of the corresponding conservative system is also shown in solid line in Figure 3(b), which has approximately the same amplitude of the master displacement $\widehat{x}_{1}$ as the corresponding forced responses. 


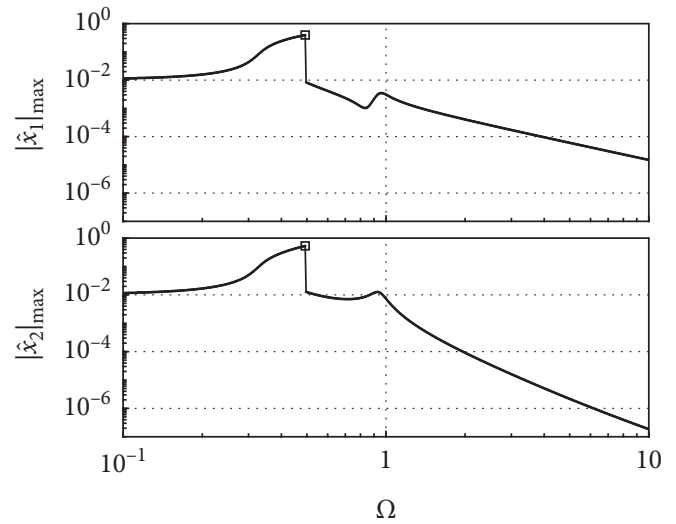

(a)

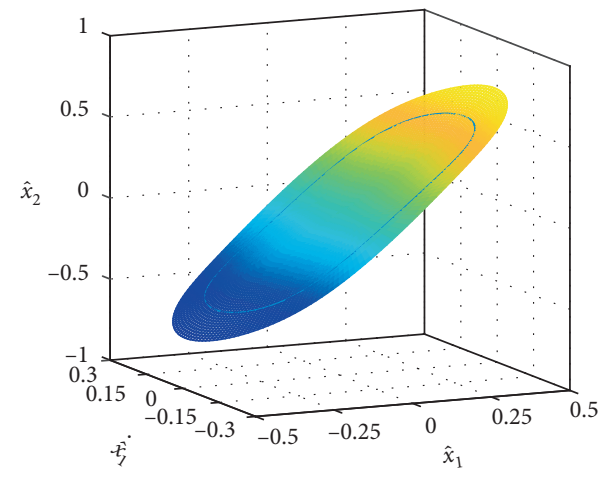

(c)

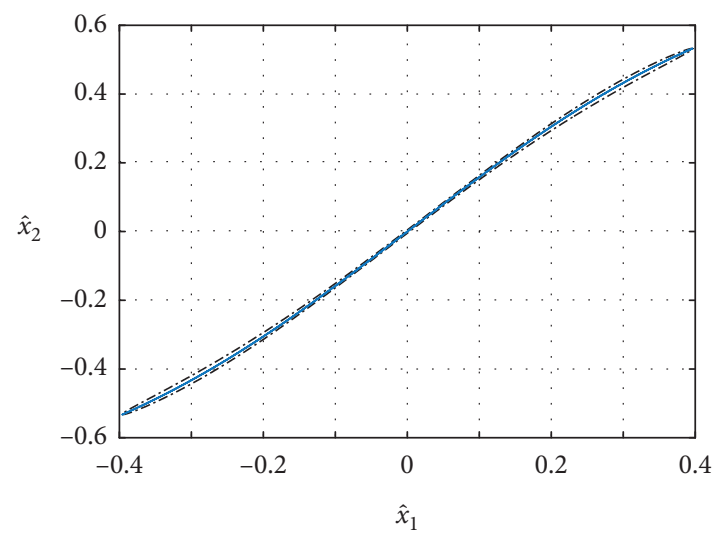

— Free periodic motion

-..-- Forced response

(b)

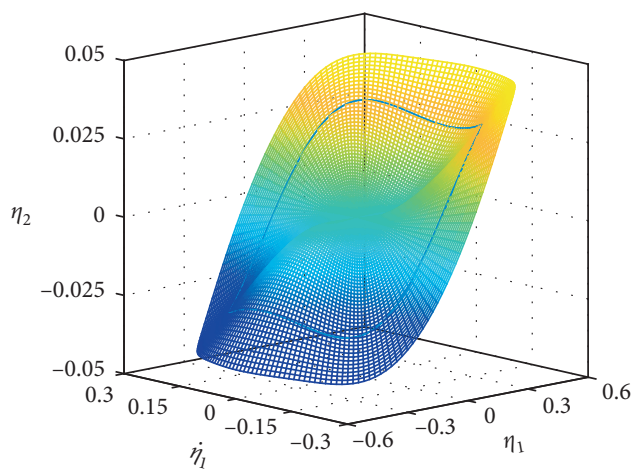

(d)

FIgURE 3: The forced responses of a type III two-stage isolator in primary resonance, for $\widehat{k}_{h 1}=1.0$ and $F=0.0015$, and the corresponding free periodic motion of NNM invariant manifold for the underlying conservative system. The other parameters of the system are the same as in Figure 2. (a) Displacement amplitude-frequency responses for the payload and the responses for the lower stage in a type III two-stage isolator. (b) The representative forced response in primary resonance of the system is shown in black dot-dashed line, which corresponds to the square points on the curves in (a). The free periodic motion of the underlying conservative system is shown in blue solid line. The corresponding forced orbit occurs in the neighbourhood of this free motion. (c) The approximation of the corresponding NNM invariant manifold for the displacement constraint $\widehat{x}_{2}\left(\widehat{x}_{1}, \dot{\hat{x}}_{1}\right)$ and the free periodic motion from (b), shown as blue solid curve. (d) The approximate NNM invariant manifold $\eta_{2}\left(\eta_{1}, \dot{\eta}_{1}\right)$ is the projection of $\hat{x}_{2}\left(\hat{x}_{1}, \dot{\hat{x}}_{1}\right)$ onto the phase space of generalized modal coordinates. The free periodic motion in (c) is also projected on this phase space as blue solid line.

As can be seen from Figures 3(a) and 3(b), two degrees of freedom in the isolator system move periodically during the significant primary resonance. The slightly distorted periodic orbit in Figure 3(b), formed by these two forced motions marked as squares in Figure 3(a), is around the corresponding free motion of the underlying conservative system, which lies on the invariant manifold surface in the phase space as shown in Figure 3(c). The invariant manifold is referred to as the nonlinear normal modes of the system.

This free periodic motion as well as the NNM invariant manifold surface can also be projected in phase subspace of modal coordinates $\eta_{1}, \dot{\eta}_{1}$, and $\eta_{2}$ in Figure 3(d). It can be seen that the nonlinear normal modes of a conservative nonlinear system can describe the coupling between different degrees of freedom or different modal oscillators of the system, as well as the forced periodic motions of the nonlinear system in primary resonance, i.e., one of the nonlinear characteristics of the response [10].

The Galerkin-based approach [14] is applied firstly here to construct a form of discretization for the governing equations of nonlinear normal modes. Then, a modified numerical scheme is presented to approximate the unknown NNM invariant manifolds, using a combination of finiteelement discretization schemes in the amplitude domain of the master coordinate $a$ and Fourier series expansions in the phase domain of the master coordinate $\phi$. For the $m$ th NNM invariant manifolds, the master coordinates $\left(\eta_{m}, \dot{\eta}_{m}\right)$ are transformed to amplitude and phase coordinates $(a, \phi)$ as follows:

$$
\begin{aligned}
& \eta_{m}(t)=a(t) \cos (\phi(t)), \\
& \dot{\eta}_{m}(t)=-\omega_{m} \cdot a(t) \sin (\phi(t)),
\end{aligned}
$$


where $\omega_{m}$ is the $m$ th natural frequency of the corresponding linear system. Substituting equation (5) into the governing equation of motion for $\eta_{m}$ in equation (4), the resulting equations governing the master coordinates $a$ and $\phi$ can be expressed as two first-order differential equations:

$$
\left\{\begin{array}{l}
\dot{a}=-\frac{f_{m}}{\omega_{m}} \sin \phi, \\
\dot{\phi}=\omega_{m}-\frac{f_{m}}{a \omega_{m}} \cos \phi .
\end{array}\right.
$$

The remaining slave coordinates on the invariant manifolds are expressed as functions of the master coordinates as follows:

$$
\begin{aligned}
\eta_{i} & =P_{i}(a, \phi), \\
\dot{\eta}_{i} & =Q_{i}(a, \phi), \\
i & =1,2, \ldots, n, i \neq m .
\end{aligned}
$$

After the substitutions of equations (7) and (6) into the equations of motion for $\eta_{i}, i \neq m$ in equation (4), the partial differential equations governing $P_{i}$ and $Q_{i}$ are generated by applying the chain rule on the manifold constraint functions $P_{i}$ and $Q_{i}$ in equation (7):

$$
\begin{gathered}
-Q_{i}+\frac{\partial P_{i}}{\partial a}\left(\frac{f_{m}}{\omega_{m}}\right) \sin \phi+\frac{\partial P_{i}}{\partial \phi}\left(\omega_{m}+\frac{f_{m} \cos \phi}{a \omega_{m}}\right)=0, \\
\omega_{i}^{2} P_{i}+f_{i}+\frac{\partial Q_{i}}{\partial a}\left(\frac{f_{m}}{\omega_{m}}\right) \sin \phi+\frac{\partial Q_{i}}{\partial \phi}\left(\omega_{m}+\frac{f_{m} \cos \phi}{a \omega_{m}}\right)=0, \\
i=1,2, \ldots, n, i \neq m .
\end{gathered}
$$

To approximate the manifold solutions to equation (8), the unknown constraint functions $P_{i}(a, \phi)$ and $Q_{i}(a, \phi)$ are expanded as a double series in $a$ and $\phi$ over a preselected amplitude-phase domain $\left\{(a, \phi) \mid a \in\left[0, a_{0}\right], \phi \in[0,2 \pi]\right\}$ as

$$
\begin{aligned}
P_{i}(a, \phi) & =\sum_{k=1}^{N_{a}} \sum_{l=1}^{N_{\phi}} C_{i}^{k, l} \cdot T_{k, l}(a, \phi), \\
Q_{i}(a, \phi) & =\sum_{k=1}^{N_{a}} \sum_{l=1}^{N_{\phi}} D_{i}^{k, l} \cdot U_{k, l}(a, \phi), \\
i & =1,2, \ldots, n, i \neq m,
\end{aligned}
$$

where the $C$ 's and $D$ 's are the unknown coefficients corresponding to the assumed shape functions $T_{k, l}(a, \phi)$ and $U_{k, l}(a, \phi)$, respectively. Substituting equation (9) into equation (8), the resulting residual functions are then projected onto the given shape functions in a Galerkin procedure as follows:

$$
\begin{array}{r}
\iint_{a, \phi} U_{p, q}\left[-a \sum_{k, l} D_{i}^{k, l} U_{k, l}+\left(\sum_{k, l} C_{i}^{k, l} \frac{\partial T_{k, l}}{\partial a}\right) \frac{f_{m}}{\omega_{m}} a \sin \phi+\left(\sum_{k, l} C_{i}^{k, l} \frac{\partial T_{k, l}}{\partial \phi}\right)\left(a \omega_{m}+\frac{f_{m} \cos \phi}{\omega_{m}}\right)\right] \cdot \mathrm{d} a \mathrm{~d} \phi=0, \\
\iint_{a, \phi} T_{p, q}\left[\omega_{i}^{2} a \sum_{k, l} C_{i}^{k, l} T_{k, l}+a f_{i}+\left(\sum_{k, l} D_{i}^{k, l} \frac{\partial U_{k, l}}{\partial a}\right) \frac{f_{m}}{\omega_{m}} a \sin \phi+\left(\sum_{k, l} D_{i}^{k, l} \frac{\partial U_{k, l}}{\partial \phi}\right)\left(a \omega_{m}+\frac{f_{m} \cos \phi}{\omega_{m}}\right)\right] \cdot \mathrm{d} a \mathrm{~d} \phi=0
\end{array}
$$

for $\quad i=1,2, \ldots, n, i \neq m ; p=1,2, \ldots, N_{a}, q=1,2, \ldots$, $N_{\phi}$.

For the unknown constraint functions $P_{i}(a, \phi)$ and $Q_{i}(a, \phi)$ in polar coordinates $(a, \phi)$, the harmonic functions are chosen naturally as expansion functions for $\phi$. In conservative dynamic systems, the choices of cosine functions in modal displacement $T_{k, l}(a, \phi)$ and sine functions in modal velocity $U_{k, l}(a, \phi)$ are applied according to the expected synchronous orbits in conservative dynamic systems $[10,14]$ :

$$
\begin{aligned}
& T_{k, l}(a, \phi)=L_{k}(a) \cos ((l-1) \phi), \\
& U_{k, l}(a, \phi)=L_{k}(a) \sin (l \phi) .
\end{aligned}
$$

A set of polynomials with orthogonality in the domain $\left[0, a_{0}\right]$ could be taken as basis functions $L_{k}(a)[14]$, resulting 

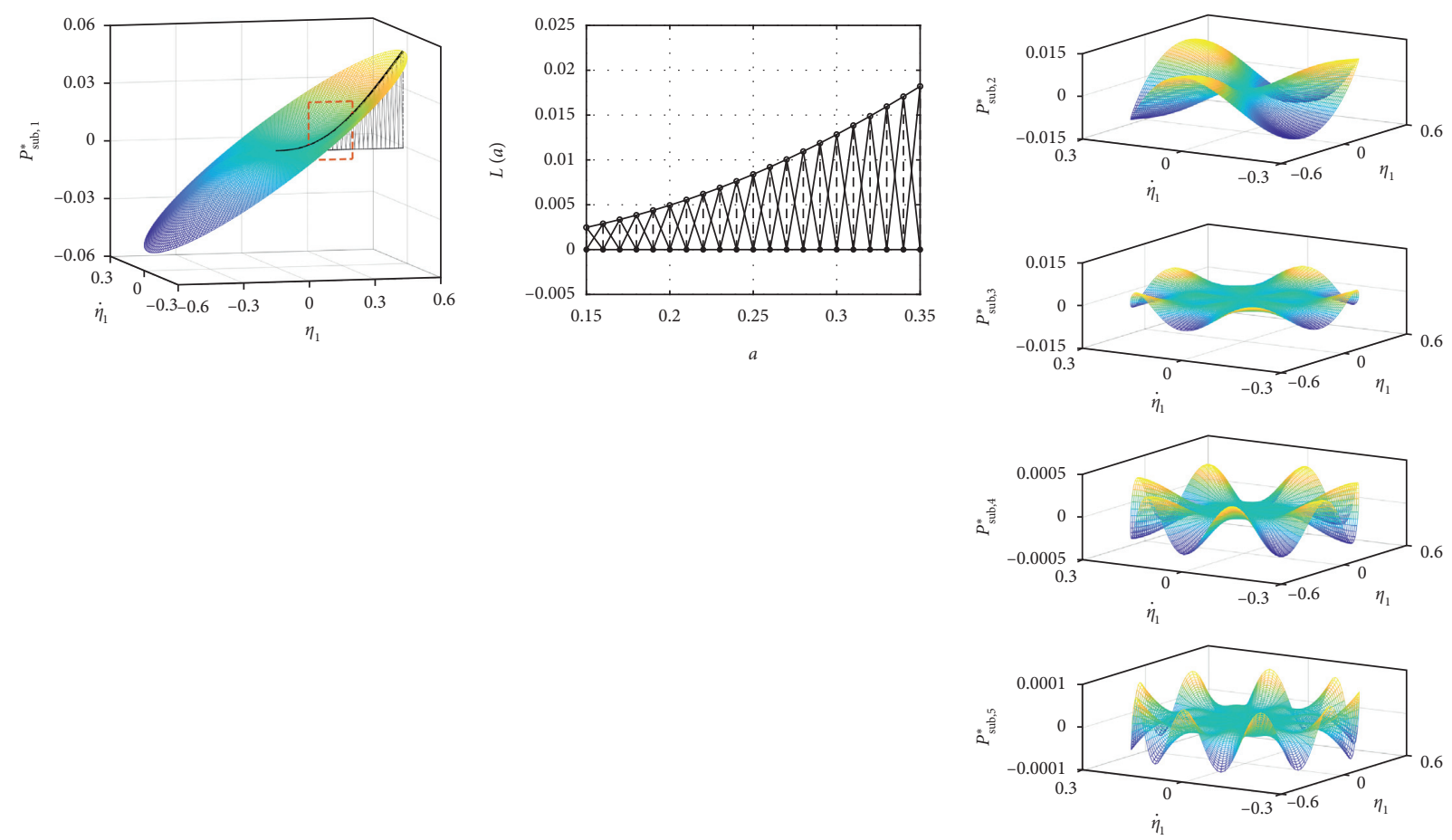

(a)

(b)

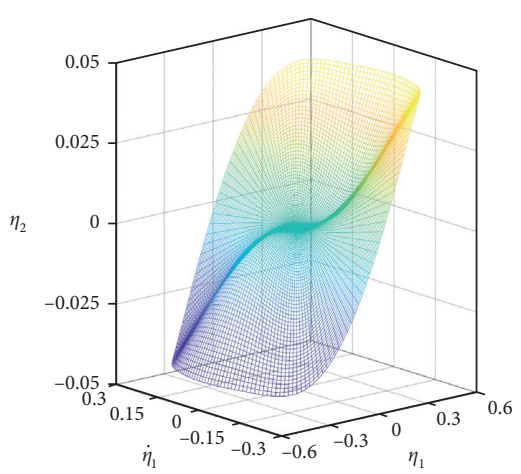

(c)

(d)

FIGURE 4: Schematic diagram of the approximate expansion of NNM invariant manifold: (a) subsurface component $P_{\text {Sub,1 }}^{*}\left(\eta_{1}, \dot{\eta}_{1}\right)$ of NNM approximation, $P_{\text {Sub }, 1}^{*}\left(\eta_{1}, \dot{\eta}_{1}\right)=P_{\text {Sub }, 1}(a, \phi)=\left(\sum_{k=1}^{N_{a}} C^{k, 1} L_{k}(a)\right) \cdot \cos \phi$; (b) piecewise linear function of finite-element approximation $L(a)$; (c) subsurface components of NNM approximation, $P_{\text {Sub }, l}^{*}\left(\eta_{1}, \dot{\eta}_{1}\right)=P_{\text {Sub }, l}(a, \phi)=\left(\sum_{k=1}^{N_{a}} C^{k, l} L_{k}(a)\right) \cdot \cos ((2 l-1) \phi)$, for $l=2, \ldots, 5 ;(\mathrm{d})$ the resultant superposition of all subsurface components, as the approximation of this NNM manifold $\eta_{2}\left(\eta_{1}, \dot{\eta}_{1}\right)$.

in a set of nonlinear algebraic equations for the unknown expansion coefficients:

$$
\begin{aligned}
& g_{i}^{p, q}\left(C_{1}^{1,1}, \ldots, C_{1}^{N_{a}, N_{\phi}} ; \ldots ; C_{j}^{1,1}, \ldots, C_{j}^{N_{a}, N_{\phi}} ; \ldots ; C_{n}^{1,1}, \ldots, C_{n}^{N_{a}, N_{\phi}} ; D_{i}^{p, q}\right) \quad=0, \\
& h_{i}^{p, q}\left(C_{1}^{1,1}, \ldots, C_{1}^{N_{a}, N_{\phi}} ; \ldots ; C_{j}^{1,1}, \ldots, C_{j}^{N_{a}, N_{\phi}} ; \ldots ; C_{n}^{1,1}, \ldots, C_{n}^{N_{a}, N_{\phi}} ; D_{i}^{1,1}, \ldots, D_{i}^{N_{a}, N_{\phi}}\right)=0 \text {, }
\end{aligned}
$$

for $p=1,2, \ldots, N_{a}, q=1,2, \ldots, N_{\phi} ; i, j=1,2, \ldots, n, i$, $j \neq m$, where $g_{i}^{p, q}=0$ and $h_{i}^{p, q}=0$ correspond to equations (10) and (11), respectively. The kind of nonlinear algebraic equations could be solved by numerical methods such as Powell's Hybrid method $[24,25]$. Note that each equation has all the coefficients $C$ s s of the expansions $P_{j}(a, \phi)$, for $j=1,2, \ldots, n, j \neq m$ due to the substitution of equation (9) into the nonlinear force terms $f_{m}$ and $f_{i}$ in equation (8) where the coefficients cannot be eliminated after the Galerkin projection. 
Unfortunately, these nonlinear algebraic equations may fail to be solved numerically with a general choice $C=0$ and $D=0$ as the initial guess, which is encountered in cases studies of this work. Instead of complicated initial guessing techniques for root finding, an alternative modified Galerkin method is applied with a combination of finiteelement discretization in the amplitude domain $a$ and Fourier series expansions in the phase domain $\phi$. After solving a set of less-coupled nonlinear algebraic equations with $C=0$ and $D=0$ as the initial guess, an accurate approximate solution of the NNM invariant manifold can be obtained.

Here, the trial functions $L(a)$ are assumed to be a set of linear functions for elements of the $a$ domain:

$$
\begin{aligned}
& L_{1}(a)= \begin{cases}\frac{a_{2}-a}{a_{2}-a_{1}}, & a \in\left[a_{1}, a_{2}\right], \\
0, & a \in\left(a_{2}, a_{N_{a}}\right]\end{cases} \\
& L_{k}(a)= \begin{cases}\frac{a-a_{k-1}}{a_{k}-a_{k-1}}, & a \in\left[a_{k-1}, a_{k}\right], \\
\frac{a_{k+1}-a}{a_{k+1}-a_{k}}, & a \in\left[a_{k}, a_{k+1}\right], \\
0, & a \in\left[a_{1}, a_{k-1}\right) \bigcup\left(a_{k+1}, a_{N_{a}},\right]\end{cases} \\
& k=2, \ldots, N_{a}-1 \text {, } \\
& L_{N_{a}}(a)= \begin{cases}0, & a \in\left[a_{1}, a_{N_{a}-1},\right) \\
\frac{a-a_{N_{a}-1}}{a_{N_{a}}-a_{N_{a}-1}}, & a \in\left[a_{N_{a}-1}, a_{N_{a}}\right],\end{cases}
\end{aligned}
$$

where $N_{a}$ represents the number of element nodes including both ends of the $a$ domain. The approximate solution of the NNM invariant manifold is given as a sum of products of the linear basis functions in $a$ and a harmonic function in $\phi$, as expressed in equations (9) and (12). According to this kind of expansion, the solution surface for an invariant manifold could be interpreted as a superposition of a series of subsurfaces. Each subsurface could be represented by a product function of $L(a)$ for a finite-element (FE) formulation and a harmonic function in this modified Galerkin method. It is exemplified in Figure 4 where a NNM manifold (corresponding to the slave modal coordinate constraint $\eta_{2}\left(\eta_{1}, \dot{\eta}_{1}\right)=P(a, \phi)$, after the coordinates transformation of equation (5)) consists of five subsurfaces approximately, i.e., $P_{\text {Sub }, l}^{*}\left(\eta_{1}, \dot{\eta}_{1}\right)=P_{\text {Sub }, l}(a, \phi)=\left(\sum_{k=1}^{N_{a}}\right.$ $\left.C^{k, l} L_{k}(a)\right) \cdot \cos ((2 l-1) \phi)$, for $l=1, \ldots, 5$. The even harmonics in the expansion have no contribution to the NNM in this case. The FE formulations $L(a)$ with equal elements, for the first subsurface $P_{\text {Sub, } 1}^{*}=\left(\sum_{k=1}^{N_{a}} C^{k, 1} L_{k}(a)\right) \cdot \cos \phi$, are also shown in the cross-section of the subsurface at $\phi=0$, i.e., parts of $L(a)$ in the red dashed box in Figure 4(a) are enlarged in Figure 4(b). The unknown coefficients in the expansion equation (9) are expressed in terms of the values of the subsurface approximation at corresponding element nodes in this approach. The basis functions for nodes in between two adjacent nodes are symmetric piecewise linear functions as expressed by equation (14b), while the basis function for each boundary point is just the half of pyramid basis function given by equations (14a) or (14c).

All the shape functions $T_{k, l}(a, \phi)$ and $U_{k, l}(a, \phi)$ themselves are chosen as test functions used in a Galerkin projection over the domain $\left\{(a, \phi) \mid a \in\left[0, a_{0}\right], \phi \in[0,2 \pi]\right\}$. Note that for each basis function $L_{k}(a)$ in $T_{k, l}(a, \phi)$ or $U_{k, l}(a, \phi)$, the node value is nonzero at only one of all the element nodes, and the function is nonzero in the neighboring element of this node, as expressed by equations (14a)-(14c). This yields a set of nonlinear algebraic equations of which each equation only has unknown coefficients corresponding to three (or two) adjacent points in every subsurface.

For $\quad p=2,3, \ldots, N_{a}-1, q=1,2, \ldots, N_{\phi} ; i, j=$ $1,2, \ldots, n, i, j \neq m$,

$$
\begin{array}{r}
g_{i}^{p, q}\left(\ldots ; C_{j}^{p-1,1}, C_{j}^{p, 1}, C_{j}^{p+1,1} ; C_{j}^{p-1,2}, C_{j}^{p, 2}, C_{j}^{p+1,2} ; \ldots ; C_{j}^{p-1, N_{\phi}}, C_{j}^{p, N_{\phi}}, C_{j}^{p+1, N_{\phi}} ; \ldots ; D_{i}^{p-1, q}, D_{i}^{p, q}, D_{i}^{p+1, q}\right)=0, \\
h_{i}^{p, q}\left(\ldots ; C_{j}^{p-1,1}, C_{j}^{p, 1}, C_{j}^{p+1,1} ; C_{j}^{p-1,2}, C_{j}^{p, 2}, C_{j}^{p+1,2} ; \ldots ; C_{j}^{p-1, N_{\phi}}, C_{j}^{p, N_{\phi}}, C_{j}^{p+1, N_{\phi}} ; \ldots ; D_{i}^{p-1,1}, D_{i}^{p, 1}, D_{i}^{p+1,1} ; D_{i}^{p-1,2}, D_{i}^{p, 2}, D_{i}^{p+1,2} ; \ldots ; D_{i}^{p-1, N_{\phi}}, D_{i}^{p, N_{\phi}}, D_{i}^{p+1, N_{\phi}}\right)=0 .
\end{array}
$$

For $p=1, q=1,2, \ldots, N_{\phi} ; i, j=1,2, \ldots, n, i, j \neq m$,

$$
\begin{array}{r}
g_{i}^{1, q}\left(\ldots ; C_{j}^{1,1}, C_{j}^{2,1} ; C_{j}^{1,2}, C_{j}^{2,2} ; \ldots ; C_{j}^{1, N_{\phi}}, C_{j}^{2, N_{\phi}} ; \ldots ; D_{i}^{1, q}, D_{i}^{2, q}\right)=0, \\
h_{i}^{1, q}\left(\ldots ; C_{j}^{1,1}, C_{j}^{2,1} ; C_{j}^{1,2}, C_{j}^{2,2} ; \ldots ; C_{j}^{1, N_{\phi}}, C_{j}^{2, N_{\phi}} ; \ldots ; D_{i}^{1,1}, D_{i}^{1,2} ; D_{i}^{1,2}, D_{i}^{2,2} ; \ldots ; D_{i}^{1, N_{\phi}}, D_{i}^{2, N_{\phi}}\right)=0 .
\end{array}
$$


For $p=N_{a}, q=1,2, \ldots, N_{\phi} ; i, j=1,2, \ldots, n, i, j \neq m$,

$$
\begin{array}{r}
g_{i}^{N_{a}, q}\left(\ldots ; C_{j}^{N_{a}-1,1}, C_{j}^{N_{a}, 1} ; C_{j}^{N_{a}-1,2}, C_{j}^{N_{a}, 2} ; \ldots ; C_{j}^{N_{a}-1, N_{\phi}}, C_{j}^{N_{a}, N_{\phi}} ; \ldots ; D_{i}^{N_{a}-1, q}, D_{i}^{N_{a}, q}\right)=0, \\
h_{i}^{N_{a}, q}\left(\ldots ; C_{j}^{N_{a}-1,1}, C_{j}^{N_{a}, 1} ; C_{j}^{N_{a}-1,2}, C_{j}^{N_{a}, 2} ; \ldots ; C_{j}^{N_{a}-1, N_{\phi}}, C_{j}^{N_{a}, N_{\phi}} ; \ldots ; D_{i}^{N_{a}-1,1}, D_{i}^{N_{a}, 1} ; D_{i}^{N_{a}-1,2}, D_{i}^{N_{a}, 2} ; \ldots ; D_{i}^{N_{a}-1, N_{\phi}}, D_{i}^{N_{a}, N_{\phi}}\right)=0 .
\end{array}
$$

In comparison with equation (13), the extent of coupling in the equations is significantly reduced since fewer coupled terms relative to the total unknowns are left in each equation of the system, which could simplify the calculation for the approximate Jacobian in the gradient-based algorithms and facilitate the numerical root findings of these equations. For the following cases in this work, all the nonlinear algebraic equations of the expansion coefficients, resulting from the modified Galerkin method, can be solved with a general choice $C=0$ and $D=0$ as initial guess.

In addition, the preselected domain for amplitude $a$ does not need to start from zero which corresponds to the origin of phase space. Figure 5 shows that the invariant manifold for an annular domain which excludes the origin of phase space can be solved directly by the proposed modified Galerkin method. Hence, the preselected amplitude domain can be set to the range where the periodic motions of nonlinear normal modes are of great interest.

A representative periodic orbit that is computed by the shooting method is given in Figures 3, 5, and 6 as a reference periodic solution of the NNM invariant manifold. In this periodic solution, the time responses for the master modal coordinate $\eta_{1}$ and the slave coordinate $\eta_{2}$ are given in Figure 6(a) as solid lines. The time responses for the physical coordinate, i.e., the displacements of the isolator's upper stage $x_{1}$ and the lower stage $x_{2}$ are also shown in Figure 6(b) as solid lines. Both responses $x_{1}(t)$ and $x_{2}(t)$ are generated after the projection of modal responses $\eta_{1}(t)$ and $\eta_{2}(t)$ onto the physical coordinates. In this case, the initial condition for this periodic solution is $\left(\eta_{1}, \dot{\eta}_{1}, \eta_{2}, \dot{\eta}_{2}\right)=$ $(0.461434348205922,0,0.023742537491393,0)$. After an approximation of invariant manifold $\eta_{2}\left(\eta_{1}, \dot{\eta}_{1}\right)$ is obtained, the system of equation (4) can be reduced to a system of single degree of freedom $\eta_{1}$ by substitution of the constraint $\eta_{2}\left(\eta_{1}, \dot{\eta}_{1}\right)$ into equation (4). Time responses for the corresponding periodic solutions are presented in Figure 6, where each solution is yielded from a different choice of manifold domain. The initial conditions for these systems of single degree of freedom are identical, i.e., $\left(\eta_{1}, \dot{\eta}_{1}\right)=$ $(0.461434348205922,0)$, which is the same as part of the initial condition of the reference periodic solution. The corresponding responses for slave coordinate $\eta_{2}$ are obtained from the substitutions of $\eta_{1}(t)$ into different constraint approximations $\eta_{2}\left(\eta_{1}, \dot{\eta}_{1}\right)$ which have different domains of master coordinates $(a, \phi)$. The physical motions $x_{1}(t)$ and $x_{2}(t)$ are generated from $\eta_{1}(t)$ and $\eta_{2}(t)$ using mode shapes of the underlying linearized system.

The responses in Figure 6 show that in this case the reference periodic solution and the solutions from different approximate invariant manifolds are nearly indistinguishable overall. The modal responses $\eta_{1}(t)$ from these different solutions are indistinguishable from the reference one even in a close-up plot. For the approximate invariant manifolds with the same mesh density, the solution from the manifold which includes the origin of the phase space is slightly closer to the reference solution than the other two solutions from annular invariant manifolds.

\section{Nonlinear Normal Modes of Type III Isolator in Different Cases}

Four cases of type III two-stage isolator with different $\widehat{k}_{h 1}$ were taken as examples to verify the validity of the proposed method. Forced response solutions of equation (2) were obtained using a fourth-order Runge-Kutta integration scheme as previous sections. The underlying conservative system of the isolator, which does not consider the damping and excitation in equation (2), could be transformed to the form of equation (4) by a modal coordinate transformation. The NNM invariant manifolds corresponding to the forced responses in primary resonance were obtained through the proposed modified Galerkin method.

In the case (i) $\widehat{k}_{h 1}=1.0$, five forced response orbits of the isolator are shown in phase subspace of physical coordinates $\widehat{x}_{1}$ and $\hat{x}_{2}$ in Figure 7(b). Each one corresponds to the peak of a displacement amplitude-frequency curve shown in Figure 7(a), representing the maximum response during a primary resonance of the system. Five values of amplitudes of the harmonic force excitations were selected in each of the four cases. Five corresponding free motions of the underlying conservative system are also presented in Figure 7(b).

\section{(i) $\widehat{k}_{h 1}=1.0$ (see Figure 7)}

Figure 7 (d) displays approximate solution surface of NNM invariant manifold for the modal coordinate constraint $\eta_{2}\left(\eta_{1}, \dot{\eta}_{1}\right)$. The number of terms for Fourier expansion in equations (9) and (13) is set to be $N_{\phi}=10$ and the domain $\{a \mid a \in[0,0.58]\}$ is divided into 58 equally sized elements with the number of nodes $N_{a}=59$, to capture the geometry of invariant manifold accurately. The corresponding surface $\hat{x}_{2}\left(\hat{x}_{1}, \dot{\hat{x}}_{1}\right)$ which is the projection of NNM invariant manifold onto the phase subspace of physical coordinates is also shown in Figure 7(c). The five free periodic trajectories in Figure 7(b), which should lie on the NNM invariant manifold, are computed numerically via the shooting method [11] as the representative reference solutions for the validation of the modified Galerkin approximation. 

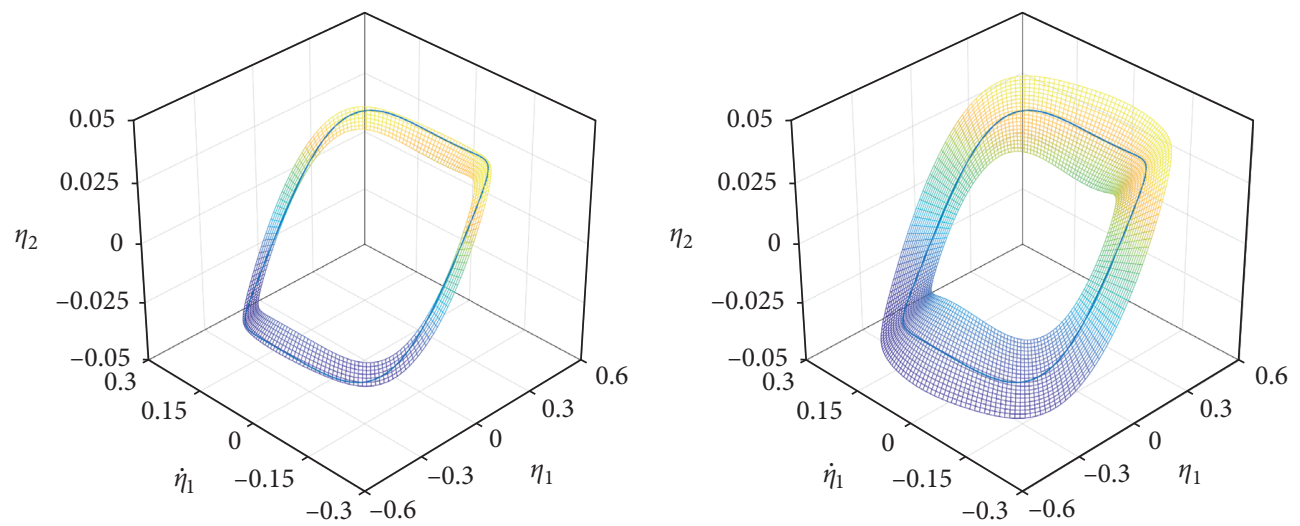

Figure 5: Two approximations of the NNM invariant manifold for the modal displacement constraint $\eta_{2}\left(\eta_{1}, \dot{\eta}_{1}\right)$ solved in respective annular domains $\{(a, \phi) \mid a \in[0.41,0.47], \phi \in[0,2 \pi]\}$ and $\{(a, \phi) \mid a \in[0.35,0.55], \phi \in[0,2 \pi]\}$ in phase subspace. The system parameters are set to the same as in Figure 3. The number of terms for Fourier expansion in equations (9) and (12) is set to be $N_{\phi}=10$, and the domain of $a$ is divided into 6 or 20 equally sized elements with the number of nodes $N_{a}=7$ or $N_{a}=21$ to accurately capture the manifold geometry. A representative periodic orbit on the manifold is shown in solid line.
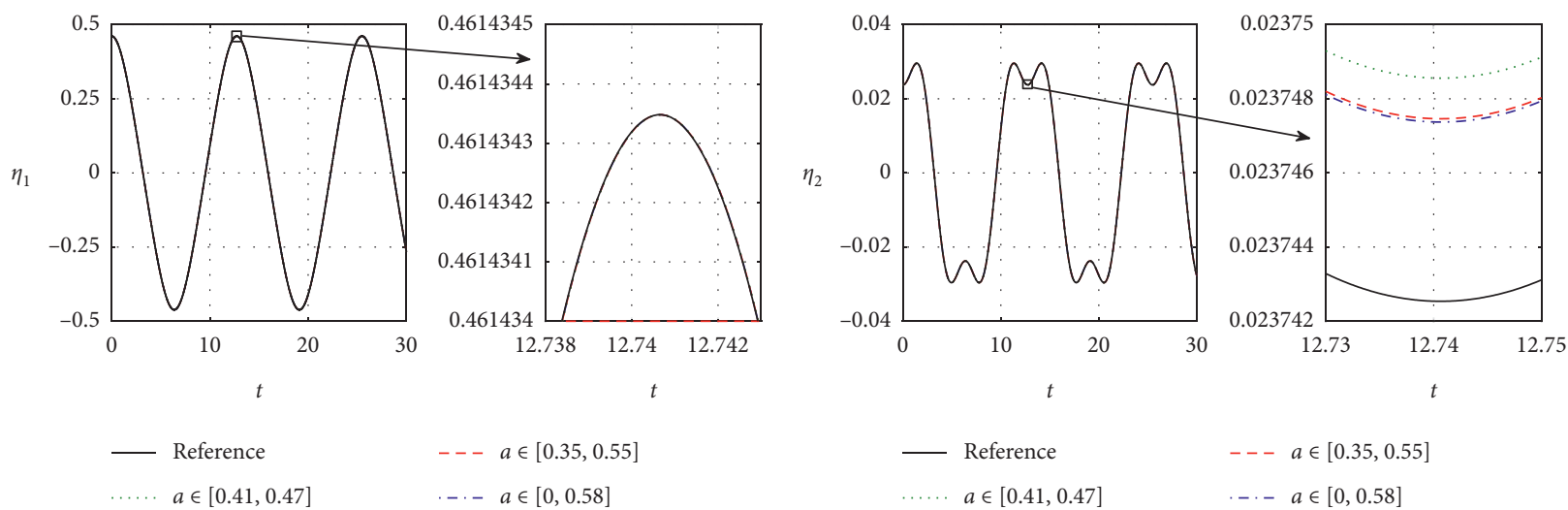

(a)
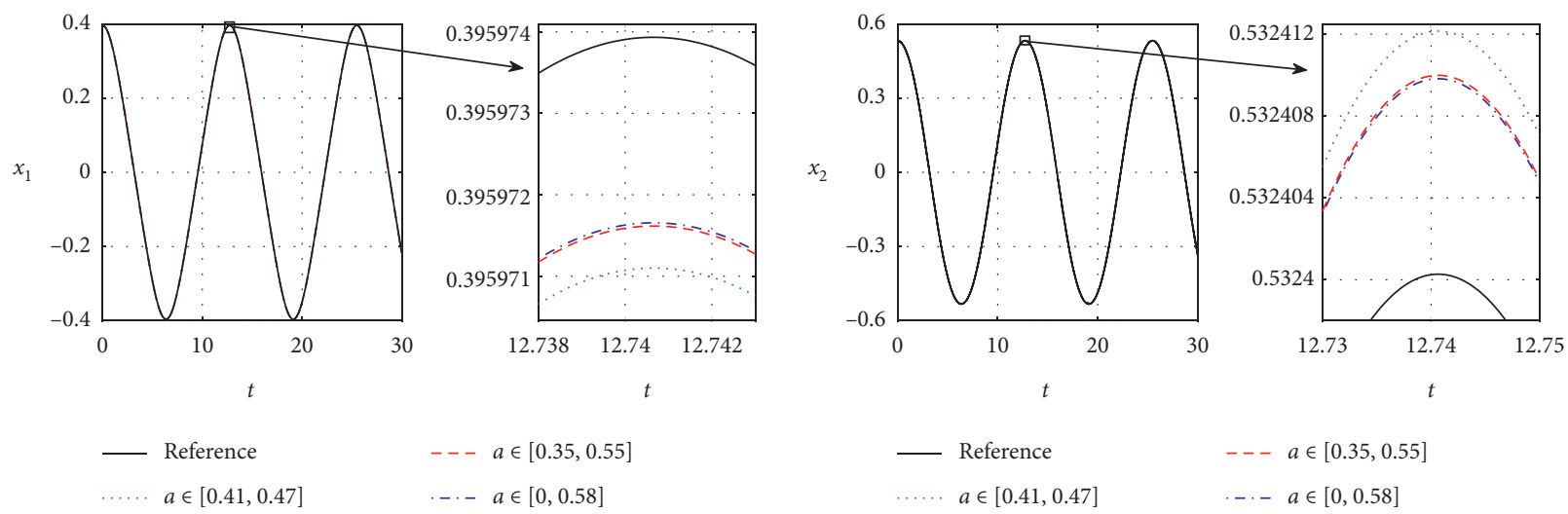

(b)

FIgURE 6: Periodic responses from various approximate invariant manifolds. Top plots: (a) response $\eta_{1}(t)$ and $\eta_{2}(t)$ for modal coordinates and the corresponding close-up plots of the square regions; bottom plots (b) response $x_{1}(t)$ and $x_{2}(t)$ for physical coordinates and the corresponding close-up plots of the square regions. Reference solution is given as solid lines; the solution from manifold of domain $\{(a, \phi) \mid a \in[0.41,0.47], \phi \in[0,2 \pi]\}$ is given as green dotted lines; the solution from manifold of domain $\{(a, \phi) \mid a \in[0.35,0.55], \phi \in[0,2 \pi]\}$ is given as red dashed lines; the solution from manifold of domain $\{(a, \phi) \mid a \in[0,0.58], \phi \in[0,2 \pi]\}$ is given as blue dashed-dotted lines. 


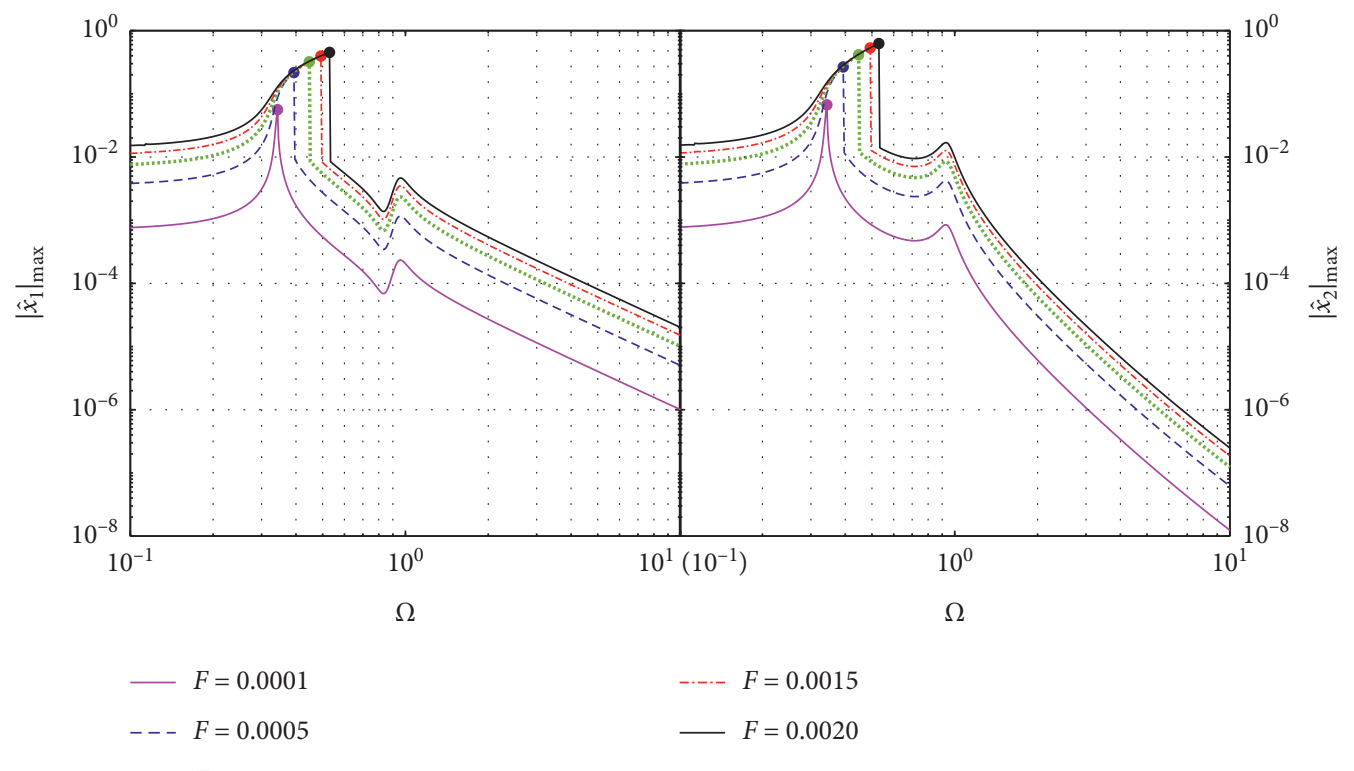

(a)

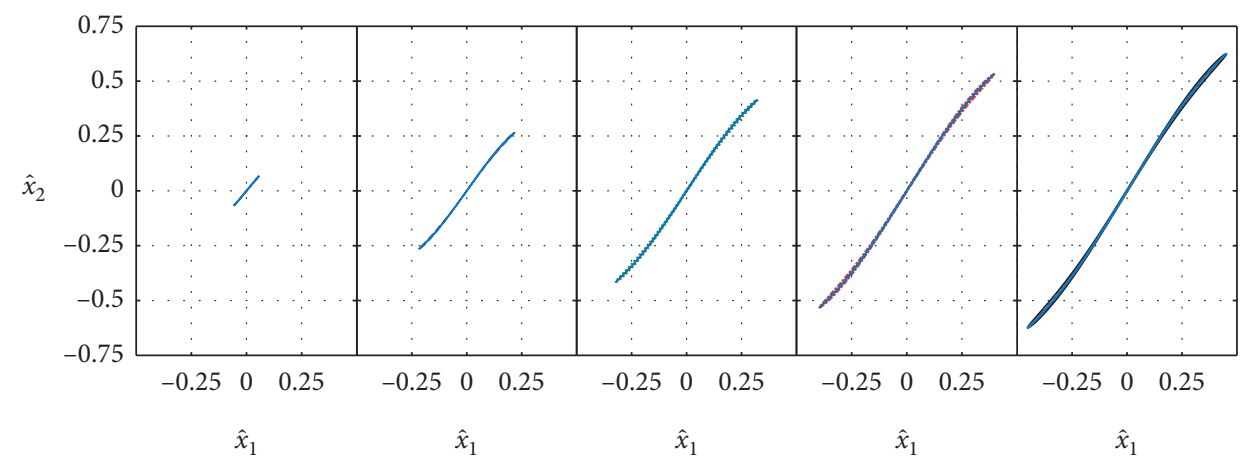

(b)

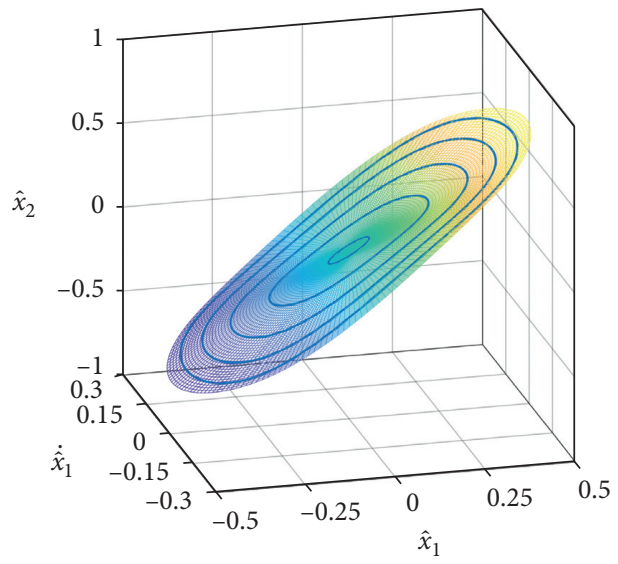

(c)

Figure 7: Continued. 


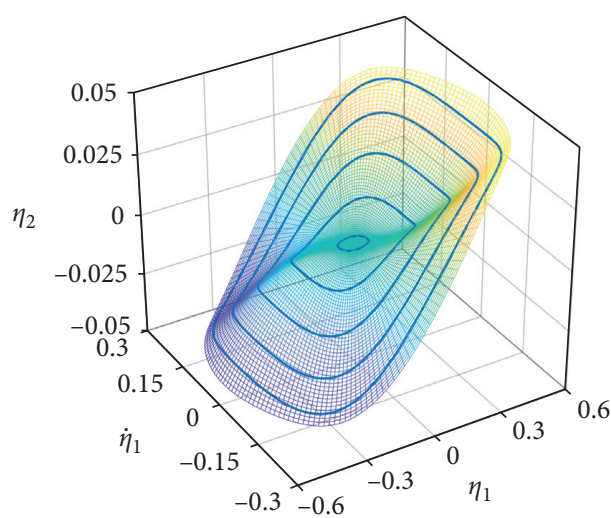

(d)

FIGURE 7: The forced responses of a type III two-stage isolator in primary resonance, for $\widehat{k}_{h 1}=1.0$, and the corresponding free periodic motions on the approximate NNM invariant manifold for the underlying conservative system. (a) Displacement amplitude-frequency responses for the payload and the ones for the lower stage of the isolator under force excitations with increasing frequency and different amplitude $F$ : pink solid line represents $F=0.0001$; blue dashed line represents $F=0.0005$; green dotted line represents $F=0.0010$; red dashed-dotted line represents $F=0.0015$; black solid line represents $F=0.0020$; the other parameters of the system are the same as in Figure 2. (b) Five representative forced responses in primary resonance of the system shown in different line styles, which correspond, respectively, to the solid points on the curves in (a) with the same color and line style. Five free periodic motions of the underlying conservative system are shown in solid lines. In the neighborhood of each periodic trajectory, the corresponding forced response orbit evolves. (c-d) The approximation of the NNM invariant manifold for the displacement constraint $\hat{x}_{2}\left(\hat{x}_{1}, \dot{\hat{x}}_{1}\right)$ and $\eta_{2}\left(\eta_{1}, \dot{\eta}_{1}\right)$, respectively; the five free periodic trajectories in (b) are also presented in solid lines in (c) and (d); $\eta_{2}\left(\eta_{1}, \dot{\eta}_{1}\right)$ is the projection of $\widehat{x}_{2}\left(\hat{x}_{1}, \dot{x}_{1}\right)$ onto the phase space of generalized modal coordinates.

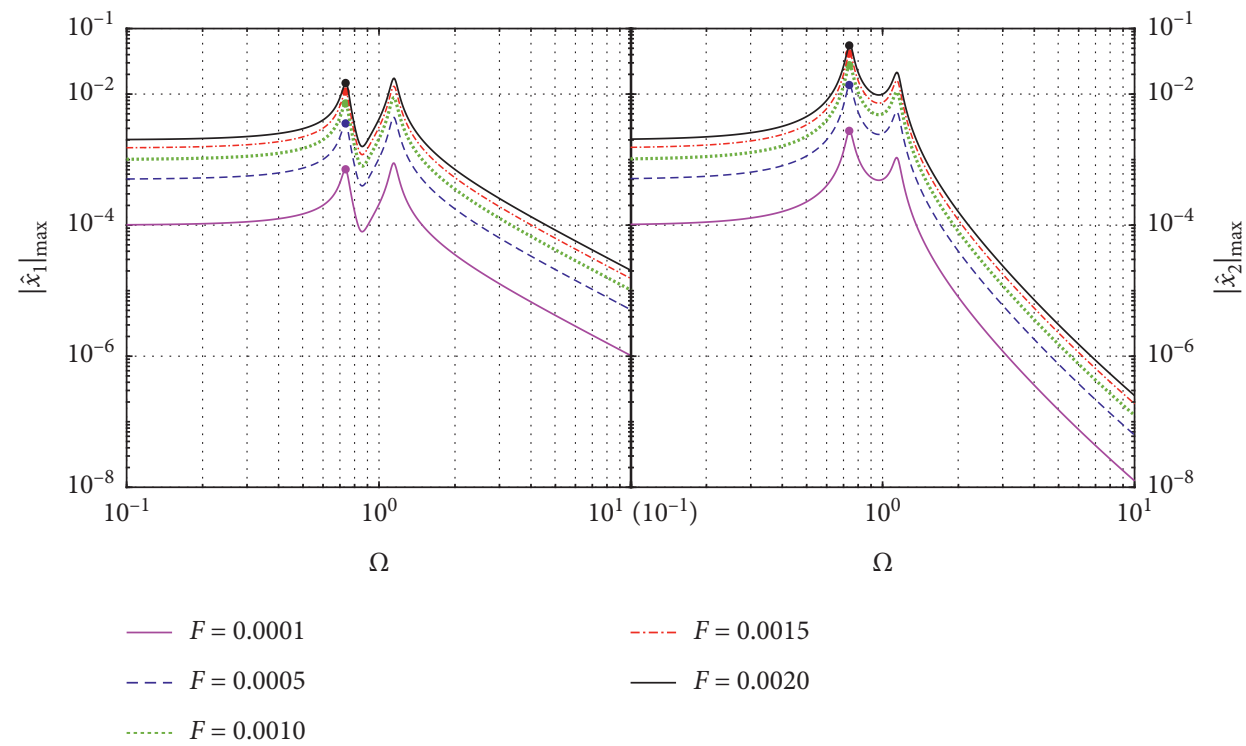

(a)

FIGURE 8: Continued. 


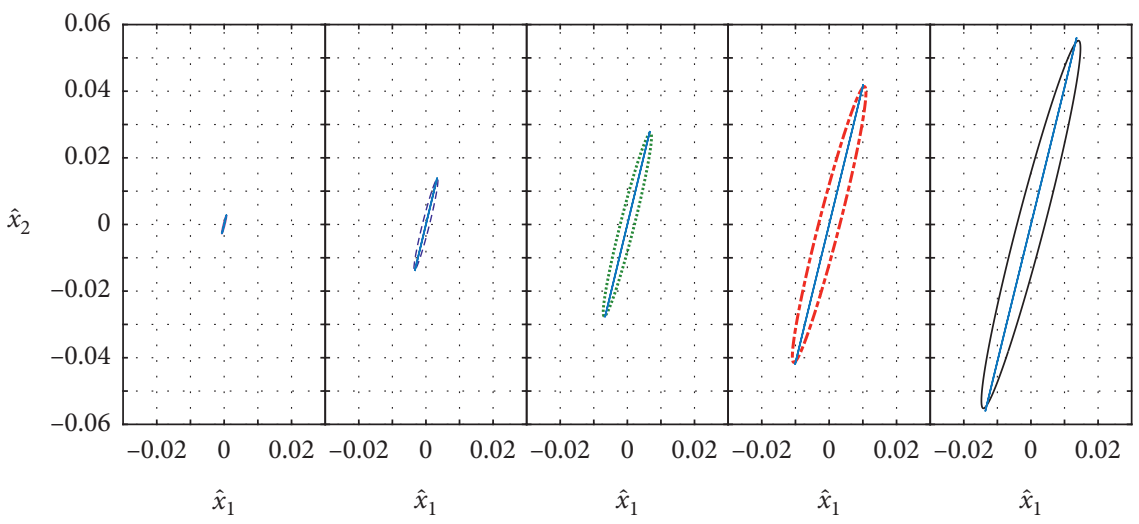

(b)

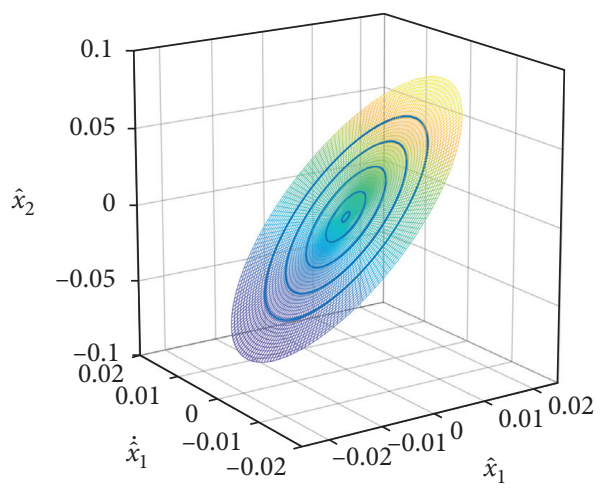

(c)

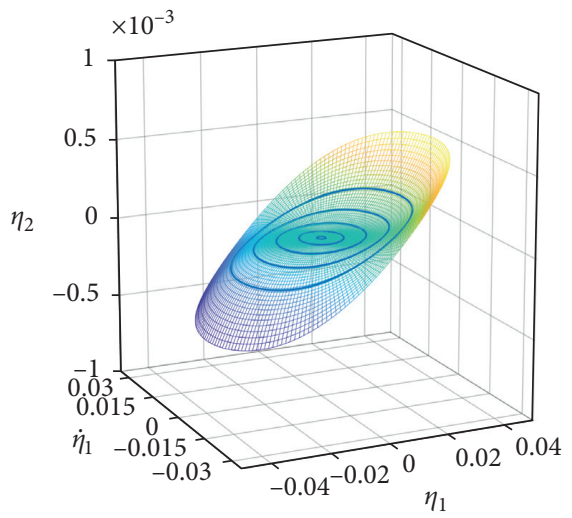

(d)

FIGURE 8: The forced responses of a type III two-stage isolator in primary resonance, for $\widehat{k}_{h 1}=0$, and the corresponding free periodic motions on the approximate NNM invariant manifold for the underlying conservative system. (a) Displacement amplitude-frequency responses for the payload and the ones for the lower stage of the isolator under harmonic force excitations with different amplitude $F$ : pink solid line represents $F=0.0001$; blue dashed line represents $F=0.0005$; green dotted line represents $F=0.0010$; red dasheddotted line represents $F=0.0015$; black solid line represents $F=0.0020$; the other system parameters are the same as in Figure 2 . (b) Five representative forced responses in primary resonance of the system shown in different line styles, which correspond, respectively, to the solid points on the curves in (a) with the same color and line style. Five corresponding free periodic trajectories of the underlying conservative system are shown in solid lines. (c-d) The approximation of the NNM invariant manifold for the displacement constraint $\hat{x}_{2}\left(\hat{x}_{1}, \dot{\hat{x}}_{1}\right)$ and $\eta_{2}\left(\eta_{1}, \dot{\eta}_{1}\right)$, respectively, and the five free periodic trajectories in (c) or (d) corresponding to the free motions in (b). To ensure the numerical accuracy, the parameters set in the numerical solution procedure are $N_{a}=41$, with 40 equally sized elements in the domain $\{a \mid a \in[0,0.04]\}$, and $N_{\phi}=6$. 


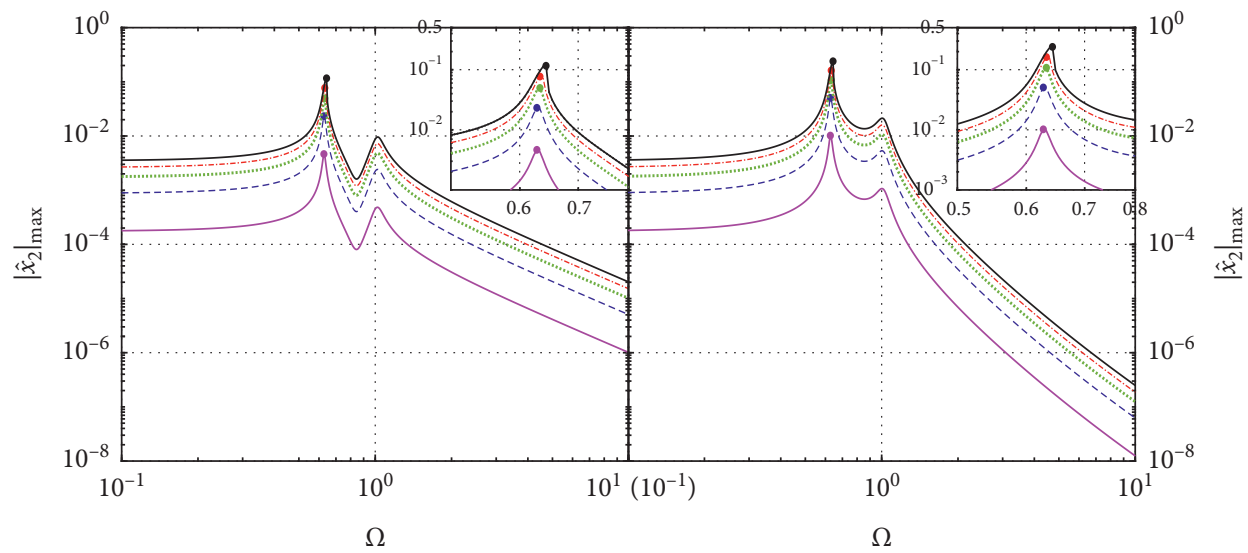
— $F=0.0001$
-.-. $F=0.0015$
- - $F=0.0005$
$-F=0.0020$
…... $F=0.0010$

(a)

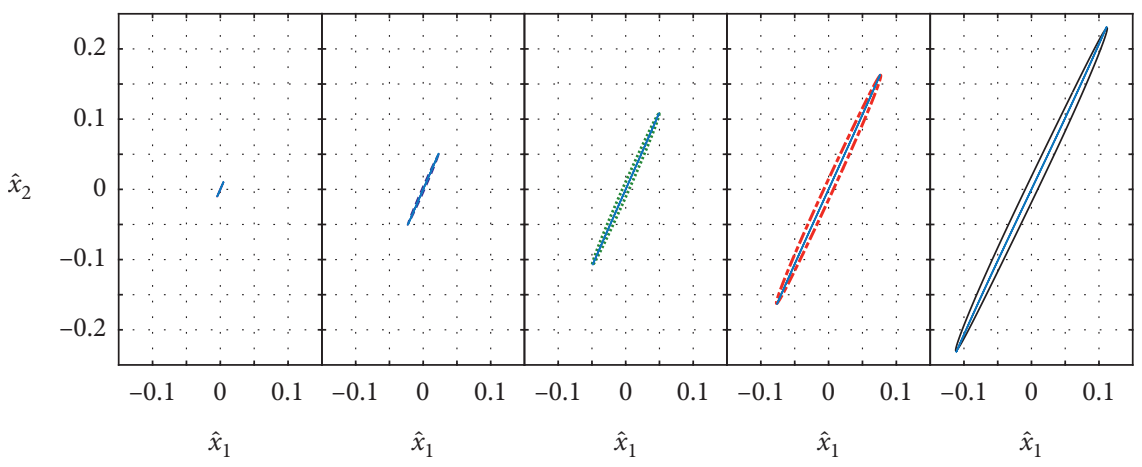

(b)

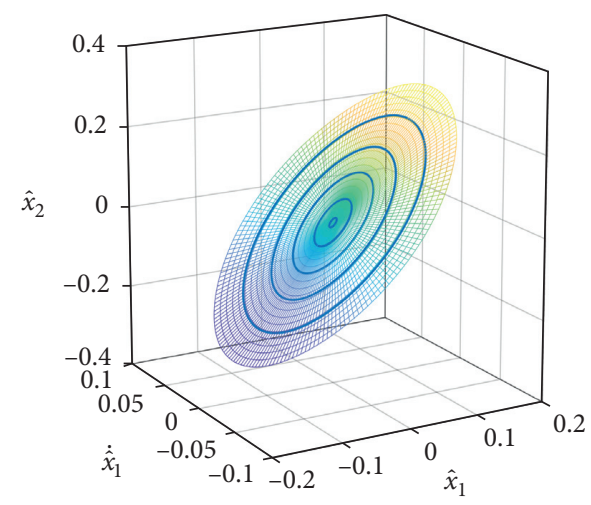

(c)

Figure 9: Continued. 


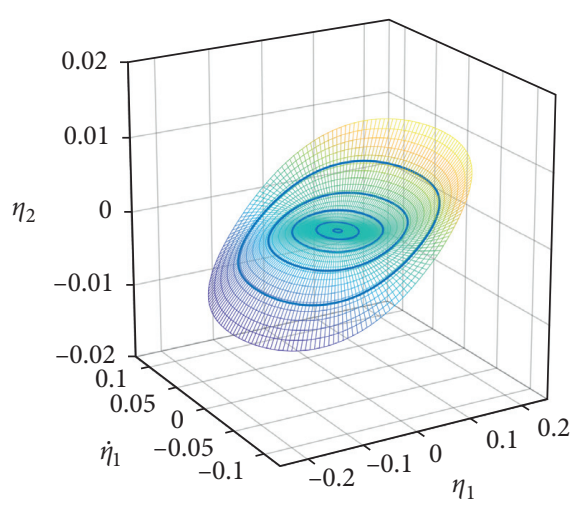

(d)

FIgURE 9: The forced responses of a type III two-stage isolator in primary resonance, for $\widehat{k}_{h 1}=0.5$, and the corresponding free periodic motions on the approximate NNM invariant manifold for the underlying conservative system. (a) Displacement amplitude-frequency responses for the payload and the ones for the lower stage of the isolator under harmonic force excitations with different amplitude $F$ : pink solid line represents $F=0.0001$; blue dashed line represents $F=0.0005$; green dotted line represents $F=0.0010$; red dashed-dotted line represents $F=0.0015$; black solid line represents $F=0.0020$. The other system parameters are the same as in Figure 2. (b) Five representative forced responses in primary resonance of the system are shown in different line styles, which correspond, respectively, to the solid points on the curves in (a) with the same color and line style. Five corresponding free periodic trajectories of the underlying conservative system are shown in solid lines, and they are also presented in (c) and (d) for different phase space. (c-d) The approximation of the NNM invariant manifold for the displacement constraint $\hat{x}_{2}\left(\hat{x}_{1}, \dot{\hat{x}}_{1}\right)$ and $\eta_{2}\left(\eta_{1}, \dot{\eta}_{1}\right)$, respectively. The parameters set in the numerical solution procedure are $N_{a}=21$, with 20 equally sized elements in the domain $\{a \mid a \in[0,0.20]\}$, and $N_{\phi}=6$.

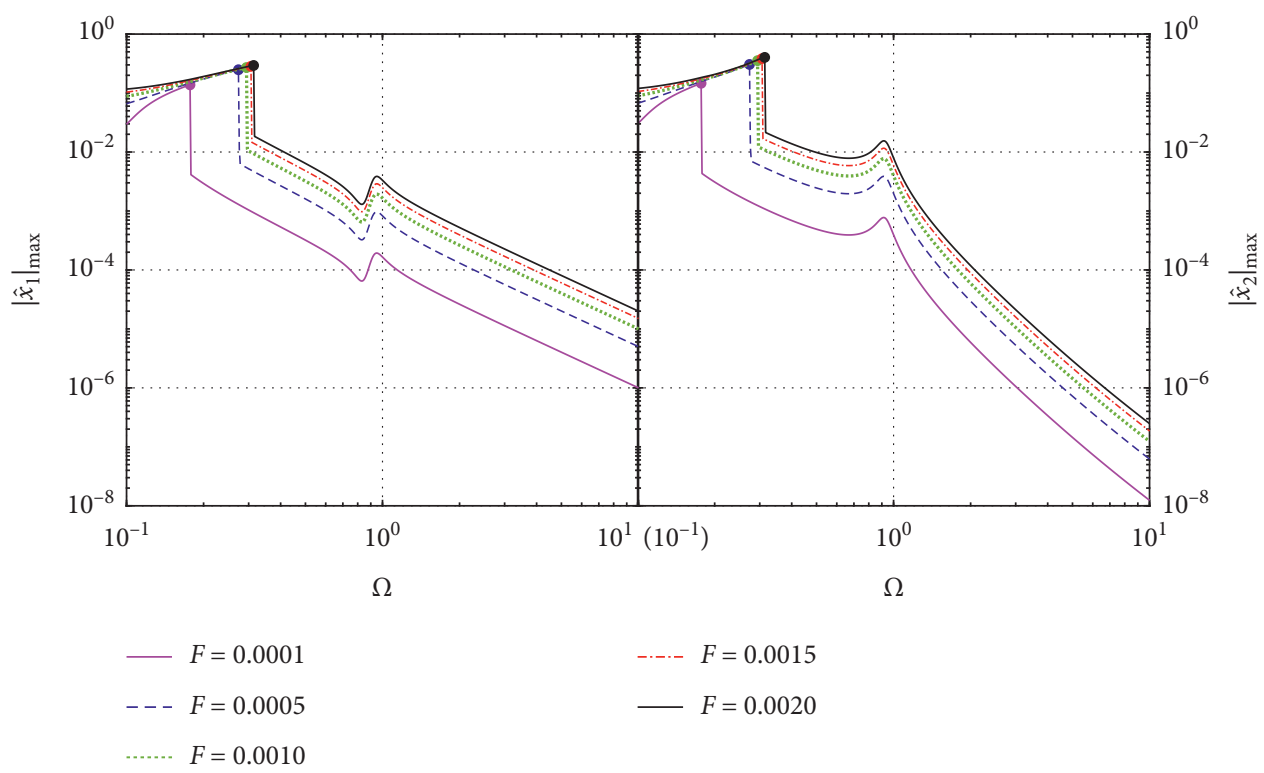

(a)

FIGURE 10: Continued. 


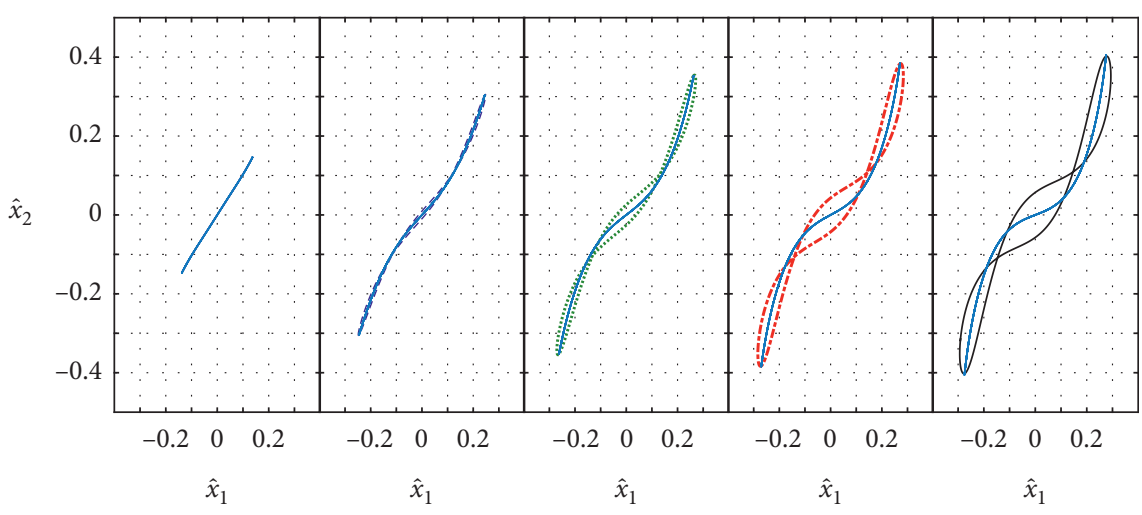

(b)

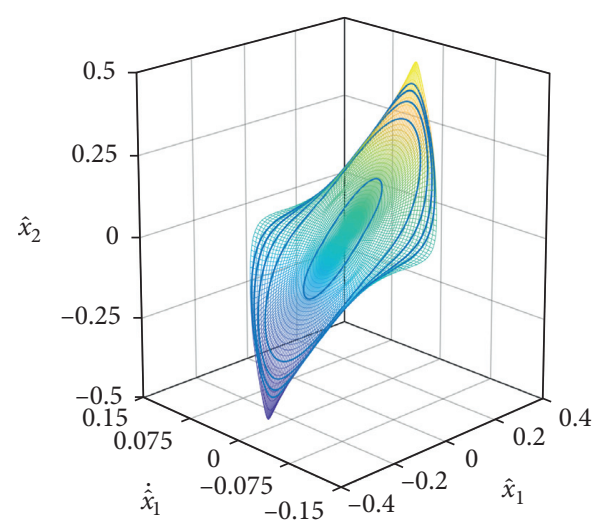

(c)

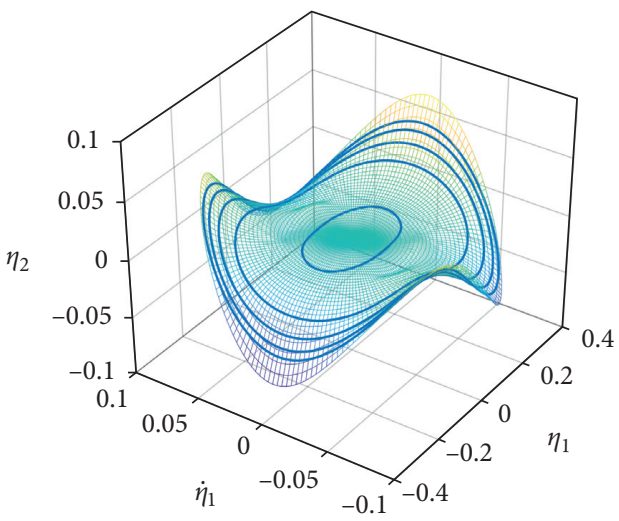

(d)

FIGURE 10: The forced responses of a type III two-stage isolator in primary resonance, for $\widehat{k}_{h 1}=1.15$, and the corresponding free periodic motions on the approximate NNM invariant manifold for the underlying conservative system. (a) Displacement amplitude-frequency responses for the payload and the ones for the lower stage of the isolator under force excitations with increasing frequency and different amplitude $F$ : pink solid line represents $F=0.0001$; blue dashed line represents $F=0.0005$; green dotted line represents $F=0.0010$; red dashed-dotted line represents $F=0.0015$; black solid line represents $F=0.0020$; the other system parameters are the same as in Figure 2. (b) Five representative forced responses in primary resonance of the system shown in different line styles, which correspond, respectively, to the solid points on the curves in (a) with the same color and line style. Five corresponding free periodic trajectories of the underlying conservative system are shown in solid lines. (c-d) The approximation of the NNM invariant manifold for the displacement constraint $\widehat{x}_{2}\left(\widehat{x}_{1}, \dot{\hat{x}}_{1}\right)$ and $\eta_{2}\left(\eta_{1}, \dot{\eta}_{1}\right)$, respectively. The parameters set in the numerical solution procedure are $N_{a}=36$, with 35 equally sized elements in the domain $\{a \mid a \in[0,0.35]\}$, and $N_{\phi}=10$. The five free periodic motions from (b) are also shown as solid curves in each phase subspace. 

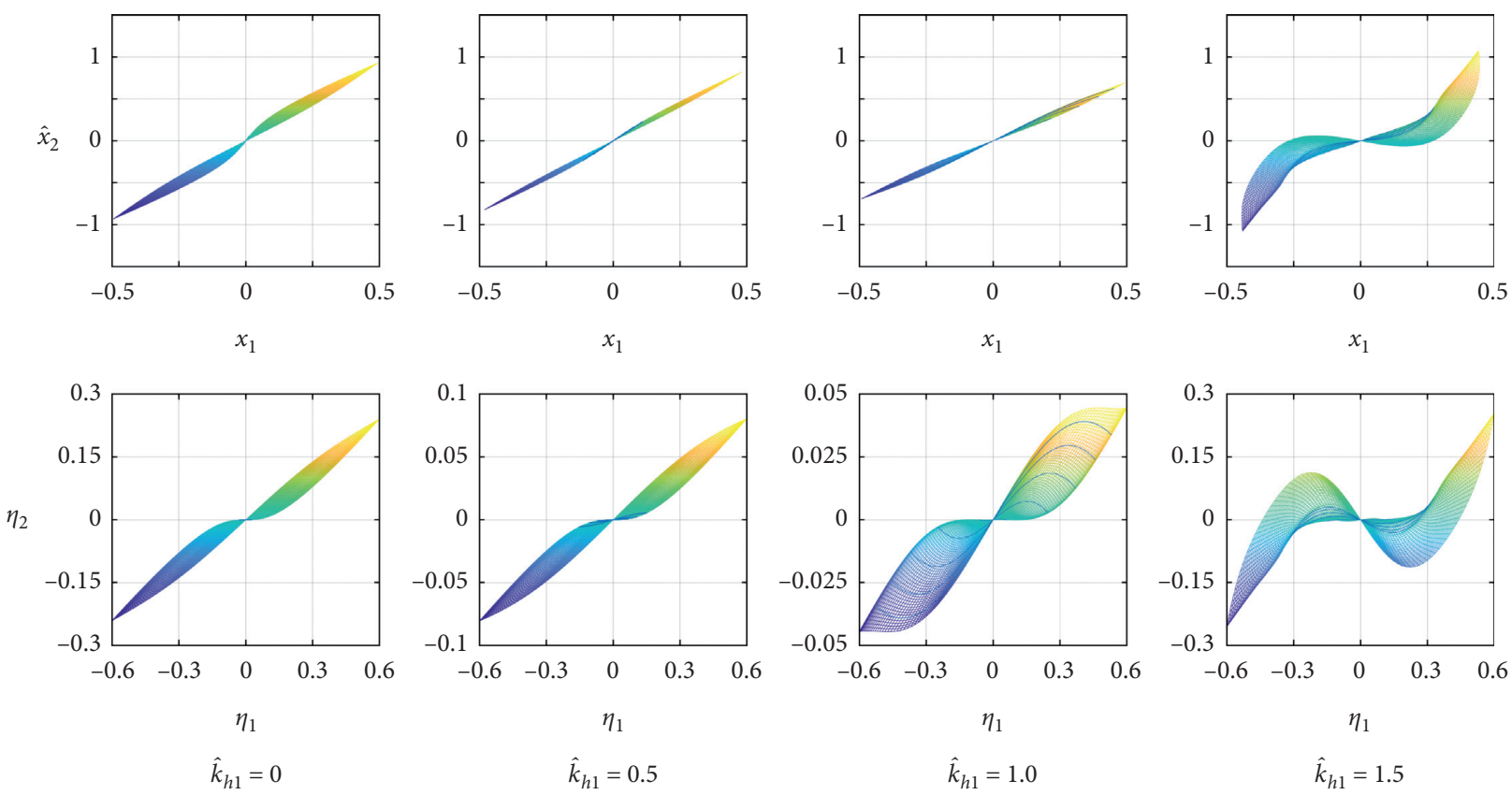

FigURE 11: Top plots: the NNM invariant manifold approximations and five representative periodic motions in the phase space of physical coordinates $\left(\widehat{x}_{1}, \widehat{x}_{2}\right)$, for $\widehat{k}_{h 1}=0, \widehat{k}_{h 1}=0.5, \widehat{k}_{h 1}=1.0$, and $\widehat{k}_{h 1}=1.15$. Bottom plots: the corresponding NNM invariant manifold approximations and periodic motions in the phase space of modal coordinates $\left(\eta_{1}, \eta_{2}\right)$, for $\widehat{k}_{h 1}=0, \widehat{k}_{h 1}=0.5, \widehat{k}_{h 1}=1.0$, and $\widehat{k}_{h 1}=1.15$.

The displayed plots in case (i) were also presented for the cases (ii) $\widehat{k}_{h 1}=0$, (iii) $\widehat{k}_{h 1}=0.5$, and (iv) $\widehat{k}_{h 1}=1.15$, respectively.

(ii) $\widehat{k}_{h 1}=0$ (see Figure 8)

(iii) $\widehat{k}_{h 1}=0.5$ (see Figure 9)

(iv) $\widehat{k}_{h 1}=1.15$ (see Figure 10)

In Figures 7(b), 8(b), 9(b), and 10(b), each reference periodic trajectory is neighbored by a corresponding forced response orbits. The information of the NNM invariant manifolds where these free periodic motions evolve can therefore give a valuable insight into the dynamics of the corresponding resonances. It can be seen from Figures 7(c), 7(d), 8(c), 8(d), 9(c), 9(d), 10(c), and 10(d) that in each case, these reference periodic trajectories agree well with the approximate manifolds obtained by the modified Galerkin method. The overall geometry of the manifold in Figures 7(d), 8(d), 9(d), or 10(d) shows apparently that, when a typical periodic motion of NNM for the system occurs, the amplitude of slave displacement $\eta_{2}$ increases nonlinearly as the amplitude of master coordinates $\eta_{1}$ increases. The NNM invariant manifold depicts the nonlinear coupling in these motions between the modal oscillators $\eta_{1}$ and $\eta_{2}$ in equation (4). The larger the amplitude of the master displacement is, the greater increment of the amplitude of the slave coordinate has. It implies that when forced periodic orbits corresponding to the NNM of the system is excited, more kinetic energy will be transferred to the slave oscillator in the period as the total energy of the system increases.
The systems for cases (i), (ii), (iii), and (iv) share a similar trend of the relationship between the amplitudes of these two modal oscillators, but the shapes of these invariant manifolds which depict the change of the coordinate relationship geometrically are different. For comparison, Figure 11 exhibits the profiles of invariant manifold approximations and reference periodic motions from the four presented cases in a phase subspace of physical coordinates $\left(\widehat{x}_{1}, \widehat{x}_{2}\right)$ and in a phase subspace of modal coordinates $\left(\eta_{1}, \eta_{2}\right)$.

Four invariant manifold profiles in modal coordinates $\left(\eta_{1}, \eta_{2}\right)$ in the bottom of Figure 11 have the same range of $\eta_{1} \in[-0.6,0.6]$. The corresponding projections of these invariant manifold profiles in physical coordinates $\left(x_{1}, x_{2}\right)$ are displayed in the top plots of Figure 11. In each invariant manifold, the slave coordinate increases nonlinearly as the master coordinate increases. The above cases also show that the stiffness component $\widehat{k}_{h 1}$ attached to the upper-stage isolator is one of the key parameters that control the degree of nonlinearity in the dynamic system. As it changes, the coupling between the oscillators of the system, depicted by synchronous periodic motions on the NNM invariant manifolds, varies accordingly. The differences in the geometries of these invariant manifolds exhibit the effect of the variation of $\widehat{k}_{h 1}$ on the nonlinear interaction between system components intuitively.

\section{Conclusions}

The proposed modified Galerkin method can be applied to accurately compute the nonlinear normal modes of a discrete dynamic system such as the new type of two-stage nonlinear isolator presented in this work. Compared with the harmonic-polynomial expansion, the applied harmonic- 
FE formulation for approximate solution yields a set of lesscoupled nonlinear algebraic equations in expansion coefficients from the standard Galerkin projection. The resulting sparser Jacobian matrix simplifies the calculation of Jacobian during the numerical root findings of the associated algebraic equations. With a general choice of zero as initial guess, these nonlinear algebraic equations generated in all the cases of this work can be solved numerically. The approximate solution of the NNM invariant manifolds can accurately capture the nonlinear interplays between the components of the conservative system in free periodic motions. When a vibrating system such as the two-stage nonlinear isolator is in primary resonance, the nonlinear coupling between the degrees of freedom can be depicted by the approximate solutions of the corresponding NNM invariant manifolds of its underlying conservative system. In addition, the invariant manifold for an annular domain of interest which excludes the origin of phase space can be solved by this approach directly.

\section{Data Availability}

The data used to support the findings of this study are available from the corresponding author upon request.

\section{Conflicts of Interest}

The authors declare that they have no conflicts of interest regarding the publication of this paper.

\section{Acknowledgments}

This work was supported by the National Natural Science Foundation of China (grant no. 11972222).

\section{References}

[1] A. Carrella, M. J. Brennan, and T. P. Waters, "Static analysis of a passive vibration isolator with quasi-zero-stiffness characteristic," Journal of Sound and Vibration, vol. 301, no. 3-5, pp. 678-689, 2007.

[2] R. A. Ibrahim, "Recent advances in nonlinear passive vibration isolators," Journal of Sound and Vibration, vol. 314, no. 3-5, pp. 371-452, 2008.

[3] Z. Lu, M. J. Brennan, T. Yang, X. Li, and Z. Liu, "An investigation of a two-stage nonlinear vibration isolation system," Journal of Sound and Vibration, vol. 332, no. 6, pp. 1456-1464, 2013.

[4] Z. Lu, T. Yang, M. J. Brennan et al., "On the performance of a two-stage vibration isolation system which has geometrically nonlinear stiffness," Journal of Vibration and Acoustics, vol. 136, no. 6, Article ID 064501, 2014.

[5] A. F. Vakakis, L. I. Manevitch, Y. V. Mikhlin, V. N. Pilipchuk, and A. A. Zevin, Normal Modes and Localization in Nonlinear Systems, Wiley Series in Nonlinear Science, Wiley, New York, NY, USA, 1996.

[6] S. W. Shaw and C. Pierre, "Non-linear normal modes and invariant manifolds," Journal of Sound and Vibration, vol. 150, no. 1, pp. 170-173, 1991.

[7] S. W. Shaw and C. Pierre, "Normal modes for non-linear vibratory systems," Journal of Sound and Vibration, vol. 164, no. 1, pp. 85-124, 1993.
[8] S. W. Shaw and C. Pierre, "Normal modes of vibration for non-linear continuous systems," Journal of Sound and Vibration, vol. 169, no. 3, pp. 319-347, 1994.

[9] J. Guckenheimer and P. Holmes, Nonlinear Oscillations, Dynamical Systems and Bifurcation of Vector Fields, Springer, New York, NY, USA, 1983.

[10] G. Kerschen, M. Peeters, J. C. Golinval, and A. F. Vakakis, "Nonlinear normal modes, Part I: a useful framework for the structural dynamicist," Mechanical Systems and Signal Processing, vol. 23, no. 1, pp. 170-194, 2009.

[11] M. Peeters, R. Viguié, G. Sérandour, G. Kerschen, and J.-C. Golinval, "Nonlinear normal modes, part II: toward a practical computation using numerical continuation techniques," Mechanical Systems and Signal Processing, vol. 23, no. 1, pp. 195-216, 2009.

[12] S. W. Shaw, C. Pierre, and E. Pesheck, "Modal analysis-based reduced-order models for nonlinear structures--an invariant manifold approach," The Shock and Vibration Digest, vol. 31, no. 1, pp. 3-16, 1999.

[13] E. Pesheck, C. Pierre, and S. W. Shaw, "Modal reduction of a nonlinear rotating beam through nonlinear normal modes," Journal of Vibration and Acoustics, vol. 124, no. 2, pp. 229236, 2002.

[14] E. Pesheck, C. Pierre, and S. W. Shaw, "A new Galerkin-based approach for accurate non-linear normal modes through invariant manifolds," Journal of Sound and Vibration, vol. 249, no. 5, pp. 971-993, 2002.

[15] E. I. Rivin, Passive Vibration Isolation, American Society of Mechanical Engineers, New York, NY, USA, 2003.

[16] M. Sun, G. Song, Y. Li, and Z. Huang, "Effect of negative stiffness mechanism in a vibration isolator with asymmetric and high-static-low-dynamic stiffness," Mechanical Systems and Signal Processing, vol. 124, pp. 388-407, 2019.

[17] G. Gatti, "A K-shaped spring configuration to boost elastic potential energy," Smart Materials and Structures, vol. 28, no. 7, 2019.

[18] G. Gatti, M. J. Brennan, and B. Tang, "Some diverse examples of exploiting the beneficial effects of geometric stiffness nonlinearity," Mechanical Systems and Signal Processing, vol. 125, pp. 4-20, 2019.

[19] A. Donmez, E. Cigeroglu, and G. O. Ozgen, "An improved quasi-zero stiffness vibration isolation system utilizing dry friction damping," Nonlinear Dynamics, vol. 101, no. 1, pp. 107-121, 2020.

[20] Z.-Q. Lu, D.-H. Gu, H. Ding, W. Lacarbonara, and L.-Q. Chen, "Nonlinear vibration isolation via a circular ring," Mechanical Systems and Signal Processing, vol. 136, Article ID 106490, 2020.

[21] Z. Lu, D. Wu, H. Ding, and L. Chen, "Vibration isolation and energy harvesting integrated in a Stewart platform with high static and low dynamic stiffness," Applied Mathematical Modelling, vol. 89, pp. 249-267, 2020.

[22] K. Ye, J. C. Ji, and T. Brown, "Design of a quasi-zero stiffness isolation system for supporting different loads," Journal of Sound and Vibration, vol. 471, Article ID 115198, 2020.

[23] F. C. Moon, Chaotic Vibrations: An Introduction for Applied Scientists and Engineers, John Wiley \& Sons, Inc., Hoboken, NJ, USA, 2004.

[24] J. J. More, B. S. Garbow, and K. E. Hillstrom, "User guide for MINPACK-1," Report ANL-80-74, Argonne National Laboratory, Argonne, IL, USA, 1980.

[25] M. J. D. Powell, Numerical Methods for Nonlinear Algebraic Equations, Gordon and Breach, London, UK, 1970. 